\title{
Asymmetric Total Synthesis of Dendrobatid Alkaloid 251F
}

Aaron Wrobleski, Kiran Sahasrabudhe, and Jeffrey Aubé*

Department of Medicinal Chemistry

University of Kansas

Lawrence, Kansas 66045-2506

\section{Supporting Information}

Experimental details and characterization data for new compounds (34 pages) 


\section{Experimental Section}

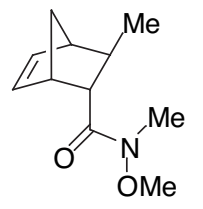

\section{3-Methyl-bicyclo[2.2.1]hept-5-ene-2-carboxylic acid methoxy-methyl-amide}

(A). Acid $4^{1}$ (4.5 g, $\left.29.6 \mathrm{mmol}\right)$ was dissolved in $125 \mathrm{~mL}$ of $\mathrm{CH}_{2} \mathrm{Cl}_{2}$ and stirred at room temperature. To this solution was added BOP (13.2 g, $29.6 \mathrm{mmol})$ and TEA (4.3 mL, $31.1 \mathrm{mmol})$. The resulting solution was stirred for $0.5 \mathrm{~h}$ upon which time $\mathrm{HNMe}(\mathrm{OMe}) \cdot \mathrm{HCl}(3.18 \mathrm{~g}, 32.6 \mathrm{mmol})$ and TEA (4.3 mL, $31.1 \mathrm{mmol})$ were added and the reaction was stirred at room temperature for $24 \mathrm{~h}$. The reaction mixture was then quenched with $1 \mathrm{~N} \mathrm{HCl}$. The aqueous layer was extracted with $\mathrm{CH}_{2} \mathrm{Cl}_{2}$, and the combined organic layers were subsequently washed with brine, dried over $\mathrm{Na}_{2} \mathrm{SO}_{4}$, filtered, concentrated, and chromatography (20\% EtOAc/hexanes) yielded the amide $(5.48 \mathrm{~g}, 95 \%$ yield $)$ as a clear, colorless oil. $[\alpha]_{\mathrm{D}}-148.2\left(\right.$ c $\left.1.50, \mathrm{CHCl}_{3}\right) ;{ }^{1} \mathrm{H} \mathrm{NMR}(500$ $\left.\mathrm{MHz} \mathrm{CDCl}_{3}\right) \delta 1.11(\mathrm{~d}, J=7.0 \mathrm{~Hz}, 3 \mathrm{H}), 1.39(\mathrm{~m}, 1 \mathrm{H}), 1.57(\mathrm{~d}, J=8.5 \mathrm{~Hz}, 1 \mathrm{H}), 1.91(\mathrm{~m}$, 1H), 2.44 (br s, 1H), 2.61 (t, $J=3.8 \mathrm{~Hz}, 1 \mathrm{H}), 3.06$ (br s, 1H), 3.12 (s, 3H), $3.68(\mathrm{~s}, 3 \mathrm{H})$, $5.89(\mathrm{dd}, J=2.8,5.6 \mathrm{~Hz}, 1 \mathrm{H}), 6.25(\mathrm{dd}, J=3.1,5.6 \mathrm{~Hz}, 1 \mathrm{H}) ;{ }^{13} \mathrm{C} \mathrm{NMR}(125 \mathrm{MHz}$, $\left.\mathrm{CDCl}_{3}\right) \delta 20.7,32.4,37.0,46.5,46.6,49.2,49.7,61.0,132.3,137.9,175.3$; IR (neat) $1670 \mathrm{~cm}^{-1}$; MS (CI) m/e $196\left(\mathrm{M}^{+}+1\right)$; HRMS calcd for $\mathrm{C}_{11} \mathrm{H}_{18} \mathrm{NO}_{2}\left(\mathrm{M}^{+}+\mathrm{H}\right):$ 196.1338, found 196.1313 .

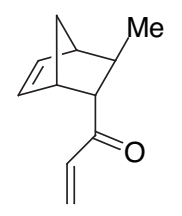

1-(3-Methyl-bicyclo[2.2.1]hept-5-en-2-yl)-propenone (5). The Weinreb amide A (980 mg, $5.03 \mathrm{mmol})$ was dissolved in $40 \mathrm{~mL}$ of $\mathrm{THF}$ and cooled to $0{ }^{\circ} \mathrm{C}$. Vinyl magnesium bromide (1.0 M in THF, $15.1 \mathrm{~mL}, 15.1 \mathrm{mmol})$ was added dropwise and the solution stirred at $0{ }^{\circ} \mathrm{C}$ for $1 \mathrm{~h}$. The reaction was then quenched with $1 \mathrm{~N} \mathrm{HCl}$, and the aqueous layer was extracted with $\mathrm{Et}_{2} \mathrm{O}$. The combined organic layers were washed with brine and dried over $\mathrm{Na}_{2} \mathrm{SO}_{4}$. Chromatography (5\% EtOAc/hexanes) yielded 5 (775 mg, 
95\% yield) as a clear, colorless oil. $[\alpha]_{\mathrm{D}}-97.9\left(c \quad 0.38, \mathrm{CHCl}_{3}\right) ;{ }^{1} \mathrm{H} \mathrm{NMR}(400 \mathrm{MHz}$, $\left.\mathrm{CDCl}_{3}\right) \delta 1.15(\mathrm{~d}, J=7.0 \mathrm{~Hz}, 3 \mathrm{H}), 1.44-1.46(\mathrm{~m}, 1 \mathrm{H}), 1.63(\mathrm{~d}, J=8.5 \mathrm{~Hz}, 1 \mathrm{H}), 1.95-1.98$ (m, 1H), 2.48 (br s, 1H), 2.67 (t, $J=4.3 \mathrm{~Hz}, 1 \mathrm{H}$ ), 3.13 (br s, $1 \mathrm{H}$ ), 5.69 (dd, $J=1.4,10.5$ $\mathrm{Hz}, 1 \mathrm{H}), 5.83$ (dd, $J=2.8,5.6 \mathrm{~Hz}, 1 \mathrm{H}), 6.20-6.25(\mathrm{~m}, 2 \mathrm{H}), 6.46$ (dd, $J=10.5,17.4 \mathrm{~Hz}$, $1 \mathrm{H}) ;{ }^{13} \mathrm{C}$ NMR $\left(100 \mathrm{MHz}, \mathrm{CDCl}_{3}\right) \delta 20.9,35.4,46.3,46.6,49.2,59.0,127.4,132.2$, 135.4, 138.5, 200.4; IR (neat) $1696 \mathrm{~cm}^{-1}$; MS (CI) m/e $163\left(\mathrm{M}^{+}+1\right)$; HRMS calcd for $\mathrm{C}_{11} \mathrm{H}_{15} \mathrm{O}\left(\mathrm{M}^{+}+\mathrm{H}\right):$ 163.1123, found 163.1113.

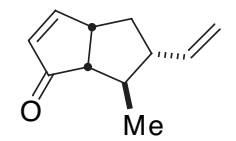

$(5 S, \quad 6 R)$-6-Methyl-5-vinyl-4,5,6,6a-tetrahydro-3a- $H$-pentalen-1-one (3). Enone 5 (200 mg, $1.23 \mathrm{mmol}, 0.01 \mathrm{M})$ was dissolved in $120 \mathrm{~mL}$ of $\mathrm{CH}_{2} \mathrm{Cl}_{2}$ and stirred at room temperature. Ethylene was bubbled through the solution for ca. 5 min followed by addition of Grubbs catalyst $(50 \mathrm{mg}, 0.06 \mathrm{mmol})$. The reaction flask was flushed with ethylene, and the reaction was allowed to stir for $12 \mathrm{~h}$ at room temperature under an ethylene balloon. The reaction was concentrated and chromatographed with 1-3\% EtOAc/hexanes to yield $\mathbf{3}(186 \mathrm{mg}, 93 \%$ yield $)$ as a clear, slightly black tinted oil. $[\alpha]_{D}$ $+182\left(c 1.13, \mathrm{CHCl}_{3}\right) ;{ }^{1} \mathrm{H} \mathrm{NMR}\left(400 \mathrm{MHz}, \mathrm{CDCl}_{3}\right) \delta 1.15(\mathrm{~d}, J=6.6 \mathrm{~Hz}, 3 \mathrm{H}), 1.18-1.27$ (m, 1H), 1.48-1.55 (m, 1H), 2.11-2.18 (m, 1H), 2.27-2.32 (m, 1H), 2.37 (dd, $J=7.0,9.1$, $1 \mathrm{H}), 3.36-3.42(\mathrm{~m}, 1 \mathrm{H}), 4.95-5.03(\mathrm{~m}, 2 \mathrm{H}), 5.55-5.64(\mathrm{~m}, 1 \mathrm{H}), 5.92(\mathrm{dd}, J=1.8,5.6,1 \mathrm{H})$, $7.58(\mathrm{dd}, J=2.6,5.6,1 \mathrm{H}) ;{ }^{13} \mathrm{C} \mathrm{NMR}\left(100 \mathrm{MHz}, \mathrm{CDCl}_{3}\right) \delta 17.6,36.4,42.2,46.3,56.5$, 57.1, 115.2, 131.2, 139.7, 166.5, 211.7; IR (neat) $1705 \mathrm{~cm}^{1}$; MS (CI) m/e $163\left(\mathrm{M}^{+}+1\right)$; HRMS calcd for $\mathrm{C}_{11} \mathrm{H}_{15} \mathrm{O}\left(\mathrm{M}^{+}+\mathrm{H}\right)$ : 163.1123 , found 163.1107.

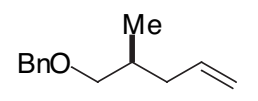

(4S)-5-Benzyloxy-4-methyl-1-pentene (B). $\mathrm{NaH}$ (785 mg, $19.6 \mathrm{mmol}, 60 \%$ in mineral oil dispersion) was washed with hexanes and then suspended in $25 \mathrm{~mL}$ of anhyrous DMF. After cooling the above suspension to $0{ }^{\circ} \mathrm{C}$, a solution of $(S)$-2-methyl4-penten-1-ol ${ }^{2}(1.63 \mathrm{~g}, 16.3 \mathrm{mmol})$ in $10 \mathrm{~mL}$ DMF was added dropwise. The mixture 
was stirred for $15 \mathrm{~min}$ at $0{ }^{\circ} \mathrm{C}$ at which time benzyl bromide $(3.62 \mathrm{~g}, 21.2 \mathrm{mmol})$ was added dropwise. The reaction was allowed to slowly warm to ambient temperature and then stirred overnight. The reaction was then quenched with water and extracted with EtOAc. The combined organic layers were washed with brine, dried over $\mathrm{Na}_{2} \mathrm{SO}_{4}$, filtered, concentrated, and chromatography (1-3\% EtOAc/hexanes) yielded B (3.09 g, $100 \%$ yield $)$ as a clear, colorless liquid. $[\alpha]_{\mathrm{D}}+2.4\left(c\right.$ 2.30, $\left.\mathrm{CHCl}_{3}\right) ;{ }^{1} \mathrm{H} \mathrm{NMR}(400 \mathrm{MHz}$, $\left.\mathrm{CDCl}_{3}\right) \delta 0.98(\mathrm{~d}, J=6.5 \mathrm{~Hz}, 3 \mathrm{H}), 1.89-2.01(\mathrm{~m}, 2 \mathrm{H}), 2.25-2.31(\mathrm{~m}, 1 \mathrm{H}), 3.32(\mathrm{dd}, J=$ 6.2, 9.1 Hz, 1H), $3.38(\mathrm{dd}, J=6.1,9.1 \mathrm{~Hz}, 1 \mathrm{H}), 4.55$ (s, 2H), 5.03-5.09 (m, 2H), 5.78$5.88(\mathrm{~m}, 1 \mathrm{H}), 7.31-7.39(\mathrm{~m}, 5 \mathrm{H}) ;{ }^{13} \mathrm{C} \mathrm{NMR}\left(100 \mathrm{MHz}, \mathrm{CDCl}_{3}\right) \delta$ 16.7, 33.3, 38.0, 72.9, 75.3, 115.9, 127.4, 127.5, 128.3, 136.9, 138.7; IR (neat) $2900 \mathrm{~cm}^{-1}$; MS (CI) m/e $191\left(\mathrm{M}^{+}\right.$ $+1)$; HRMS calcd for $\mathrm{C}_{13} \mathrm{H}_{19} \mathrm{O}\left(\mathrm{M}^{+}+\mathrm{H}\right)$ : 191.1436, found 191.1446.

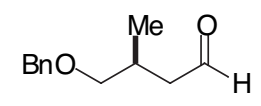

(3S)-4-Benzyloxy-3-methyl-butyraldehyde (7). (4S)-5-Benzyloxy-4-methyl-1pentene $(\mathbf{B}, 3.09 \mathrm{~g}, 16.3 \mathrm{mmol})$ was diluted in $100 \mathrm{~mL}$ of $1: 1$ ether/water and stirred at ambient temperature. To this solution was added $\mathrm{OsO}_{4}(125 \mathrm{mg}, 0.49 \mathrm{mmol})$ dissolved in $25 \mathrm{~mL}$ of water followed by NMNO $(2.86 \mathrm{~g}, 24.5 \mathrm{mmol})$. The reaction was stirred for 5 min and $\mathrm{NaIO}_{4}(5.24 \mathrm{~g}, 24.5 \mathrm{mmol})$ was then added in one portion. After stirring for 24 $\mathrm{h}$, the reaction was filtered thru Celite, and the aqueous layer was extracted with ether. The combined organic layers were washed with brine, dried over $\mathrm{Na}_{2} \mathrm{SO}_{4}$, filtered, concentrated, and chromatography (5-10\% EtOAc/hexanes) yielded 7 (2.20 g, $70 \%$ yield) as a clear, colorless liquid. $[\alpha]_{\mathrm{D}}-11.2\left(\right.$ c $\left.1.70, \mathrm{CHCl}_{3}\right) ;{ }^{1} \mathrm{H} \mathrm{NMR}\left(400 \mathrm{MHz}, \mathrm{CDCl}_{3}\right)$ $\delta 1.01(\mathrm{~d}, J=6.8 \mathrm{~Hz}, 3 \mathrm{H}), 2.27-2.34(\mathrm{~m}, 1 \mathrm{H}), 2.43-2.48(\mathrm{~m}, 1 \mathrm{H}), 2.55-2.61(\mathrm{~m}, 1 \mathrm{H}), 3.27$ $(\mathrm{dd}, J=7.6,9.1 \mathrm{~Hz}, 1 \mathrm{H}), 3.44(\mathrm{dd}, J=5.1,9.1 \mathrm{~Hz}, 1 \mathrm{H}), 4.51(\mathrm{~s}, 2 \mathrm{H}), 7.31-7.39(\mathrm{~m}, 5 \mathrm{H})$, $9.79(\mathrm{t}, J=2.2 \mathrm{~Hz}, 1 \mathrm{H}) ;{ }^{13} \mathrm{C} \mathrm{NMR}\left(100 \mathrm{MHz} \mathrm{CDCl}_{3}\right) \delta 17.0,29.0,48.4,73.0,74.8$, 127.50, 127.53, 128.3, 138.2, 202.4; IR (neat) 2750, $1725 \mathrm{~cm}^{-1}$; MS (CI) m/e $193\left(\mathrm{M}^{+}+\right.$ 1); HRMS calcd for $\mathrm{C}_{12} \mathrm{H}_{17} \mathrm{O}_{2}\left(\mathrm{M}^{+}+\mathrm{H}\right): 193.1229$, found 193.1241 . 


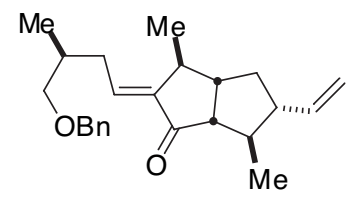

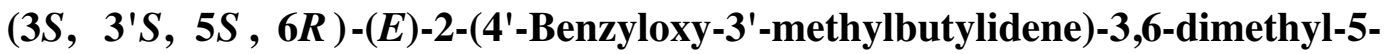
vinyl-hexahydro-penten-1-one (8). CuI (295 mg, $1.54 \mathrm{mmol})$ was suspended in $20 \mathrm{~mL}$ of THF. The mixture was cooled to $0{ }^{\circ} \mathrm{C}$, and $\mathrm{MeLi}\left(1.4 \mathrm{M}^{\text {in }} \mathrm{Et}_{2} \mathrm{O}, 2.20 \mathrm{~mL}, 3.09\right.$ mmol) was added dropwise, causing the solution to initially form a heterogeneous yellow solution that ultimaltely turned clear with complete addition of MeLi. Enone 3 (200 mg, $1.23 \mathrm{mmol}$ ), diluted in $1 \mathrm{~mL}$ of THF, was added to the above cuprate solution dropwise, again forming a yellow, heterogeneous solution. The reaction mixture was stirred for 20 min at $0{ }^{\circ} \mathrm{C}$ followed by addition of aldehyde $7(235 \mathrm{mg}, 1.23 \mathrm{mmol})$ in $1 \mathrm{ml}$ of THF. The resulting solution was stirred for $15 \mathrm{~min}$ at $0{ }^{\circ} \mathrm{C}, 15 \mathrm{~min}$ at room temperature, and then quenched with saturated $\mathrm{NH}_{4} \mathrm{Cl}$. The reaction was stirred at room temperature for ca. $1 \mathrm{~h}$. The aqueous layer was extracted with $\mathrm{Et}_{2} \mathrm{O}$, and the combined organic layers were washed with brine, dried over $\mathrm{Na}_{2} \mathrm{SO}_{4}$, filtered, concentrated, and chromatography (1-3\% EtOAc/hexanes) afforded 8 (325 mg, 60\% yield) as a clear, colorless oil. $[\alpha]_{\mathrm{D}}$ $+60.0\left(c 0.850, \mathrm{CHCl}_{3}\right) ;{ }^{1} \mathrm{H} \mathrm{NMR}\left(400 \mathrm{MHz} \mathrm{CDCl}_{3}\right) \delta 0.96(\mathrm{~d}, J=6.6 \mathrm{~Hz}, 3 \mathrm{H}), 0.98-$ $1.08(\mathrm{~m}, 1 \mathrm{H}), 1.12(\mathrm{~d}, J=7.2 \mathrm{~Hz}, 3 \mathrm{H}), 1.18$ (d, $J=6.8 \mathrm{~Hz}, 3 \mathrm{H}), 1.77-1.80$ (m, 1H), 1.99 $2.11(\mathrm{~m}, 4 \mathrm{H}), 2.35-2.47(\mathrm{~m}, 3 \mathrm{H}), 2.75(\mathrm{q}, J=7.1 \mathrm{~Hz}, 1 \mathrm{H}), 3.31-3.39(\mathrm{~m}, 2 \mathrm{H}), 4.52(\mathrm{~s}$, 2H), 4.93-5.02 (m, 2H), 5.59-5.67 (m, 1H), $6.61(\mathrm{dt}, J=1.5,6.5 \mathrm{~Hz}, 1 \mathrm{H}), 7.28-7.37$ (m, $5 \mathrm{H}) ;{ }^{13} \mathrm{C}$ NMR $\left(100 \mathrm{MHz}, \mathrm{CDCl}_{3}\right) \delta 16.8,19.9,21.6,33.2,33.6,36.6,40.4,44.1,46.1$, $54.1,58.3,73.0,75.1,114.4,127.5,128.3,136.8,138.4,140.6,143.3,209.8$; IR (neat) $1709 \mathrm{~cm}^{-1}$; MS (CI) m/e $353\left(\mathrm{M}^{+}+1\right)$; HRMS calcd for $\mathrm{C}_{24} \mathrm{H}_{33} \mathrm{O}_{2}\left(\mathrm{M}^{+}+\mathrm{H}\right): 353.2481$, found 353.2467 .
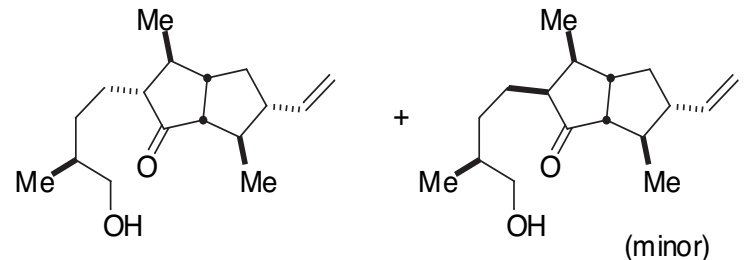

$(2 R, 3 S, 3 ' S, 5 S, 6 R)-2-\left(4^{\prime}-\right.$ Hydroxy-3'-methylbutyl)-3,6-dimethyl-5-vinylhexahydro-penten-1-one $(\mathbf{C})$. Ammonia $(15 \mathrm{~mL})$ was condensed into a 3-necked round 
bottom flask fitted with a cold-finger condensor. To the flask was added $15 \mathrm{~mL}$ of THF and absolute ethanol $(0.28 \mathrm{~mL}, 4.73 \mathrm{mmol})$, and the solution was stirred at $-78{ }^{\circ} \mathrm{C}$. Pieces of sodium were added to this solution until a dark blue color was maintained for 5 min. Enone 8 (330 $\mathrm{mg}, 0.95 \mathrm{mmol})$ in $3 \mathrm{~mL}$ of $\mathrm{THF}$ was added to the above solution dropwise. If the dark blue color dissipated, more sodium was added until the reaction mixture was again dark blue. The addition of enone was continued, adding more sodium when necessary. Upon complete addition of $\mathbf{8}$, the dark blue reaction was allowed to stir for an additional $5 \mathrm{~min}$ at which time it was quenched with saturated $\mathrm{NH}_{4} \mathrm{Cl}$. The ammonia was allowed to evaporate, and the aqueous layer was extracted with $\mathrm{Et}_{2} \mathrm{O}$. The combined organic layers were washed with brine, dried over $\mathrm{Na}_{2} \mathrm{SO}_{4}$, filtered, concentrated, and chromatographed (5-10\% EtOAc/hexanes) to yield $\mathbf{C}$ (134 $\mathrm{mg}, 54 \%$ yield) as a clear, colorless oil as an ca. 4:1 mixture of diastereomers. Major isomer $\mathbf{C}$ : ${ }^{1} \mathrm{H}$ NMR (400 MHz, $\left.\mathrm{CDCl}_{3}\right) \delta 0.91(\mathrm{~d}, J=6.7 \mathrm{~Hz}, 3 \mathrm{H}), 1.06(\mathrm{~d}, J=6.4 \mathrm{~Hz}, 3 \mathrm{H}), 1.10(\mathrm{~d}$, $J=6.4 \mathrm{~Hz}, 3 \mathrm{H}), 1.24-1.28$ (m, 2H), 1.49-1.63 (m, 6H), 1.84-1.87 (m, 2H), 2.11-2.28 (m, $4 \mathrm{H}), 3.43-3.52(\mathrm{~m}, 2 \mathrm{H}), 4.94-5.03(\mathrm{~m}, 2 \mathrm{H}), 5.58-5.67(\mathrm{~m}, 1 \mathrm{H}) ;{ }^{13} \mathrm{C}$ NMR $(100 \mathrm{MHz}$, $\left.\mathrm{CDCl}_{3}\right) \delta 16.5,17.1,18.6,24.0,29.8,35.8,38.0,41.8,44.1,46.3,55.2,58.8,59.6,67.6$, 114.8, 140.7, 220.3; IR (neat) 3420, $1726 \mathrm{~cm}^{-1}$; MS (CI) m/e $265\left(\mathrm{M}^{+}+1\right)$; HRMS calcd for $\mathrm{C}_{17} \mathrm{H}_{29} \mathrm{O}_{2}\left(\mathrm{M}^{+}+\mathrm{H}\right): 265.2168$, found 265.2178. Minor diastereomer ${ }^{13} \mathrm{C}$ NMR (100 $\left.\mathrm{MHz}, \mathrm{CDCl}_{3}\right) \delta 16.9,18.5,21.2,22.6,31.0,31.5,35.7,39.4,45.8,45.9,49.4,54.6,56.9$, $67.7,115.0,140.0,221.6$.

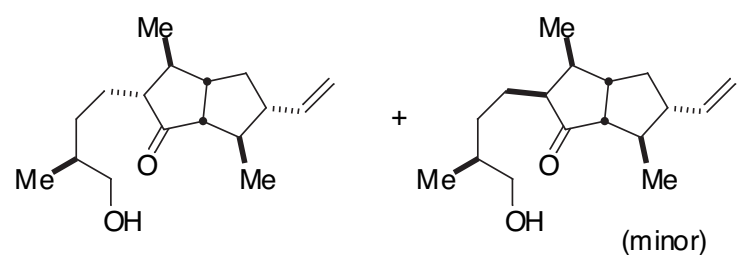

$(2 R, 3 S, 3 ' S, 5 S, 6 R)-2-\left(4^{\prime}\right.$-Azido-3'-methylbutyl)-3,6-dimethyl-5-vinylhexahydro-penten-1-one (9). Compound C (226 mg, $0.86 \mathrm{mmol})$ was diluted in $25 \mathrm{~mL}$ of anhydrous toluene and stirred at room temperature. To this solution was added $\mathrm{Zn}\left(\mathrm{N}_{3}\right) 2 \cdot 2$ pyr (396 mg, $\left.1.28 \mathrm{mmol}\right)$ and triphenylphosphine $(913 \mathrm{mg}, 3.42 \mathrm{mmol})$. To the above heterogeneous solution was added DEAD $(0.565 \mathrm{~mL}, 3.42 \mathrm{mmol})$, and the reaction was allowed to stir for $12 \mathrm{~h}$ at room temperature. The reaction was quenched with water, and the aqueous layer was extracted with $\mathrm{Et}_{2} \mathrm{O}$. The combined organic layers 
were washed with brine, dried over $\mathrm{Na}_{2} \mathrm{SO}_{4}$, filtered, concentrated, and chromatographed (5\% EtOAc/hexanes) to yield 9 (245 mg, 100\% yield) as a clear, colorless oil. Major isomer: ${ }^{1} \mathrm{H} \mathrm{NMR}\left(500 \mathrm{MHz}, \mathrm{CDCl}_{3}\right) \delta 1.00(\mathrm{~d}, J=6.4 \mathrm{~Hz}, 3 \mathrm{H}), 1.10(\mathrm{~d}, J=6.4 \mathrm{~Hz}, 3 \mathrm{H})$, $1.14(\mathrm{~d}, J=6.3 \mathrm{~Hz}, 3 \mathrm{H}), 1.28-1.39(\mathrm{~m}, 3 \mathrm{H}), 1.51-1.59$ (m, 4H), 1.69-1.74 (m, 1H), $1.87-$ $1.91(\mathrm{~m}, 1 \mathrm{H}), 2.15-2.34(\mathrm{~m}, 4 \mathrm{H}), 3.15(\mathrm{dd}, J=6.8,11.9 \mathrm{~Hz}, 1 \mathrm{H}), 3.25(\mathrm{dd}, J=5.9,12.0$ $\mathrm{Hz}, 1 \mathrm{H}), 4.99-5.06(\mathrm{~m}, 2 \mathrm{H}), 5.63-5.70(\mathrm{~m}, 1 \mathrm{H}) ;{ }^{13} \mathrm{C} \mathrm{NMR}\left(125 \mathrm{MHz}, \mathrm{CDCl}_{3}\right) \delta 17.2$, 17.6, 18.7, 24.6, 31.1, 33.9, 39.5, 42.0, 44.3, 46.4, 55.2, 57.6, 58.5, 59.7, 114.8, 140.7, 219.6; IR (neat) 2095, $1726 \mathrm{~cm}^{-1}$; MS (CI) m/e $262\left(\mathrm{M}^{+}+1\right.$ - 28). Minor diastereomer ${ }^{13} \mathrm{C} \mathrm{NMR}\left(125 \mathrm{MHz}, \mathrm{CDCl}_{3}\right) \delta 16.9,17.7,18.5,21.5,32.1,33.8,35.6,39.5,45.9,46.0$, $49.3,54.6,56.9,57.4,115.0,140.0,221.0$.

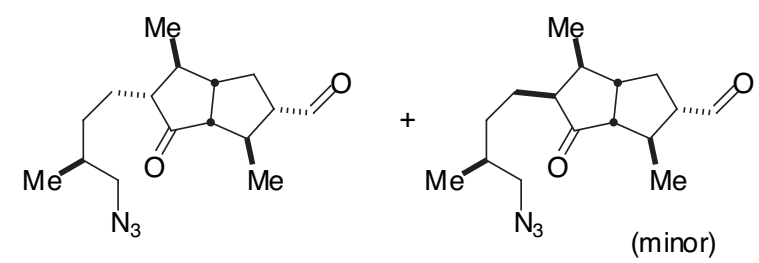

$(1 R, 2 S, 3 ' S, 4 S, 5 R)-5$-(4'-Azido-3'-methylbutyl)-1,4-dimethyl-6-oxooctahydro-pentalene-2-carbaldehyde (D). Azide 9 (130 mg, $0.45 \mathrm{mmol})$ was diluted in $4 \mathrm{~mL}$ of anydrous $\mathrm{CH}_{2} \mathrm{Cl}_{2}$ and cooled to $-78^{\circ} \mathrm{C}$. Ozone was bubbled through the solution until a blue tint was observed (ca. $3 \mathrm{~min}$ ). To the resulting blue solution was added $3 \mathrm{~mL}$ of dimethyl sulfide. The reaction was allowed to warm to ambient temperature and stirred for 16-24 $\mathrm{h}$ at which point it was concentrated and chromatographed (5\% EtOAc/hexanes) to yield $\mathbf{D}\left(92 \mathrm{mg}, 70 \%\right.$ yield) as a clear colorless oil. Major isomer: ${ }^{1} \mathrm{H}$ $\operatorname{NMR}\left(500 \mathrm{MHz}, \mathrm{CDCl}_{3}\right) \delta 0.96(\mathrm{~d}, J=6.2 \mathrm{~Hz}, 3 \mathrm{H}), 1.14(\mathrm{~d}, J=6.4 \mathrm{~Hz}, 3 \mathrm{H}), 1.20(\mathrm{~d}, J=$ $6.6 \mathrm{~Hz}, 3 \mathrm{H}), 1.47-1.56(\mathrm{~m}, 4 \mathrm{H}), 1.66-1.79(\mathrm{~m}, 3 \mathrm{H}), 1.85-1.89(\mathrm{~m}, 1 \mathrm{H}), 2.15-2.33(\mathrm{~m}, 3 \mathrm{H})$, $2.38(\mathrm{t}, J=9.4 \mathrm{~Hz}, 1 \mathrm{H}), 2.50-2.56(\mathrm{~m}, 1 \mathrm{H}), 3.13(\mathrm{dd}, J=6.8,12.0 \mathrm{~Hz}, 1 \mathrm{H}), 3.21(\mathrm{dd}, J=$ 5.9, $12.0 \mathrm{~Hz}, 1 \mathrm{H}), 9.61(\mathrm{~d}, J=2.8 \mathrm{~Hz}, 1 \mathrm{H}) ;{ }^{13} \mathrm{C} \mathrm{NMR}\left(125 \mathrm{MHz}, \mathrm{CDCl}_{3}\right) \delta 17.5,18.4$, 18.6, 24.6, 30.9, 31.0, 33.8, 37.7, 42.8, 47.1, 57.4, 58.1, 59.5, 61.4, 202.4, 218.1; IR (neat) 2720, 2090, $1721 \mathrm{~cm}^{-1}$; MS (CI) $m / e 264\left(\mathrm{M}^{+}+1-28\right)$. Minor diastereomer ${ }^{13} \mathrm{C}$ NMR $\left(125 \mathrm{MHz}, \mathrm{CDCl}_{3}\right) \delta$ 16.6, 17.6, 19.7, 21.6, 31.9, 33.2, 33.7, 35.6, 41.0, 46.4, 49.7, $56.8,57.3,61.6,202.3,220.0$. 


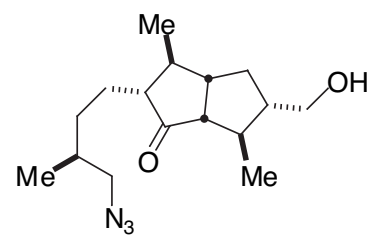

$(2 R, \quad 3 S, \quad 3 ' S, \quad 5 S, \quad 6 R)$-2-(4'-Azido-3'-methylbutyl)-3,6-dimethyl-5hydroxymethyl-hexahydro-penten-1-one (2): Compound D (92 mg, $0.32 \mathrm{mmol})$ was diluted in $5 \mathrm{~mL}$ of $\mathrm{EtOH}$ and cooled to $0{ }^{\circ} \mathrm{C}$. Sodium borohydride $(12 \mathrm{mg}, 0.32 \mathrm{mmol})$ was dissolved in ca. $1 \mathrm{~mL}$ of EtOH and added dropwise to the above solution. TLC analysis of the reaction mixture immediately after borohydride addition indicated consumption of all starting material. The reaction was quenched with $1 \mathrm{~N} \mathrm{HCl}$, stirred for $10 \mathrm{~min}$, and concentrated. The aqueous layer was extracted with $\mathrm{CH}_{2} \mathrm{Cl}_{2}$, and the combined organic layers were washed with brine, dried over $\mathrm{Na}_{2} \mathrm{SO}_{4}$, filtered, concentrated and chromatographed (3-10\% EtOAc/hexanes) to yield 2 (72 $\mathrm{mg}, 78 \%$ yield) as a clear, colorless oil. $[\alpha]_{\mathrm{D}}+11.9\left(\right.$ c 2.10, $\left.\mathrm{CHCl}_{3}\right) ;{ }^{1} \mathrm{H} \mathrm{NMR}\left(400 \mathrm{MHz}, \mathrm{CDCl}_{3}\right)$ $\delta 0.96(\mathrm{~d}, J=6.7 \mathrm{~Hz}, 3 \mathrm{H}), 1.12(\mathrm{t}, J=6.8 \mathrm{~Hz}, 6 \mathrm{H}), 1.30-1.36(\mathrm{~m}, 1 \mathrm{H}), 1.47-1.57(\mathrm{~m}$, 4H), 1.63-1.86 (m, 6H), 2.15-2.30 (m, 3H), $3.12(\mathrm{dd}, J=6.8,12.0 \mathrm{~Hz}, 1 \mathrm{H}), 3.21$ (dd, $J=$ $5.9,12.0 \mathrm{~Hz}, 1 \mathrm{H}), 3.53(\mathrm{dd}, J=6.4,10.7 \mathrm{~Hz}, 1 \mathrm{H}), 3.70(\mathrm{dd}, J=4.4,10.6 \mathrm{~Hz}, 1 \mathrm{H}) ;{ }^{13} \mathrm{C}$ NMR $\left(100 \mathrm{MHz} \mathrm{CDCl}_{3}\right) \delta 17.5,18.1,18.6,24.7,31.0,33.8,34.2,38.4,43.5,46.2,51.9$, 57.5, 58.1, 60.1 , 64.6, 219.9; IR (neat) 3430, 2095, $1726 \mathrm{~cm}^{-1}$; MS (CI) m/e $266\left(\mathrm{M}^{+}+1\right.$ 28).

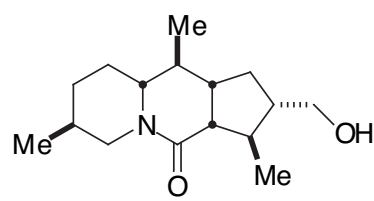

6-Oxo-251F (10). Azide 2 (90 mg, $0.31 \mathrm{mmol}$ ) was diluted in $10 \mathrm{~mL}$ of $\mathrm{CH}_{2} \mathrm{Cl}_{2}$ and cooled to $0{ }^{\circ} \mathrm{C}$. Triflic acid $(0.135 \mathrm{~mL}, 1.54 \mathrm{mmol})$ was added dropwise and stirred at $0{ }^{\circ} \mathrm{C}$ until TLC indicated the consumption of all starting material (ca. $6 \mathrm{~h}$ ). The reaction was then quenched with saturated $\mathrm{NaHCO}_{3}$. The aqueous layer was extracted with $\mathrm{CH}_{2} \mathrm{Cl}_{2}$, and the combined organic layers were washed with brine, dried over 
$\mathrm{Na}_{2} \mathrm{SO}_{4}$, filtered, concentrated and chromatographed (30-100\% EtOAc/hexanes) to yield 10 (64 mg, 79\% yield) as a clear, oily solid. Crystallization from $\mathrm{Et}_{2} \mathrm{O}$ at $0{ }^{\circ} \mathrm{C}$ gave crystals suitable for X-ray analysis. $[\alpha]_{\mathrm{D}}+28.9\left(c 1.00, \mathrm{CHCl}_{3}\right)$; ${ }^{1} \mathrm{H}$ NMR $(400 \mathrm{MHz}$, $\left.\mathrm{CDCl}_{3}\right) \delta 0.87(\mathrm{~d}, J=6.6 \mathrm{~Hz}, 3 \mathrm{H}), 0.95(\mathrm{~d}, J=6.4 \mathrm{~Hz}, 3 \mathrm{H}), 0.98-1.07(\mathrm{~m}, 2 \mathrm{H}), 1.20(\mathrm{~d}, J$ $=6.2 \mathrm{~Hz}, 3 \mathrm{H}), 1.22-1.26(\mathrm{~m}, 1 \mathrm{H}), 1.38-1.42(\mathrm{~m}, 2 \mathrm{H}), 1.71-1.84(\mathrm{~m}, 4 \mathrm{H}), 1.93(\mathrm{t}, J=12.3$ $\mathrm{Hz}, 1 \mathrm{H}), 2.00-2.08(\mathrm{~m}, 2 \mathrm{H}), 2.18-2.23(\mathrm{~m}, 2 \mathrm{H}), 2.66(\mathrm{dt}, J=2.5,9.7 \mathrm{~Hz}, 1 \mathrm{H}), 3.47$ (dd, $J$ $=6.6,10.5 \mathrm{~Hz}, 1 \mathrm{H}), 3.68(\mathrm{dd}, J=4.3,10.6 \mathrm{~Hz}, 1 \mathrm{H}), 4.64-4.69(\mathrm{~m}, 1 \mathrm{H}) ;{ }^{13} \mathrm{C} \mathrm{NMR}(100$ $\left.\mathrm{MHz}, \mathrm{CDCl}_{3}\right) \delta 16.5,18.6,19.1,30.5,32.5,32.7$ (2C), 38.1, 40.3, 40.8, 48.7, 48.8, 52.3, 61.8, 65.2, 171.3; IR (neat) 3455, $1615 \mathrm{~cm}^{-1}$; MS (CI) m/e $266\left(\mathrm{M}^{+}+1\right)$; HRMS calcd for $\mathrm{C}_{16} \mathrm{H}_{28} \mathrm{NO}_{2}\left(\mathrm{M}^{+}+\mathrm{H}\right): 266.2120$, found 266.2146.

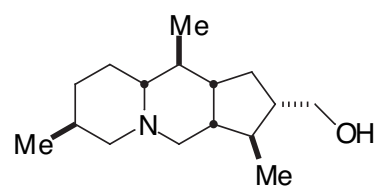

251F (1): Lactam $10(111 \mathrm{mg}, 0.42 \mathrm{mmol})$ was dissolved in $10 \mathrm{~mL}$ of anhydrous $\mathrm{Et}_{2} \mathrm{O}$ and cooled to $0{ }^{\circ} \mathrm{C}$. To this solution was added $\mathrm{LAH}\left(1.0 \mathrm{M}\right.$ in $\mathrm{Et}_{2} \mathrm{O}, 0.840 \mathrm{~mL}$, $0.84 \mathrm{mmol}$ ) dropwise. After $20 \mathrm{~min}$ at $0{ }^{\circ} \mathrm{C}$, TLC indicated no trace of lactam starting material. The reaction was quenched with $30 \mu \mathrm{L}$ water, followed by $30 \mu \mathrm{L}$ of $15 \% \mathrm{KOH}$, and finally an additional $90 \mu \mathrm{L}$ of water. The solution was stirred for $10 \mathrm{~min}$ at $0{ }^{\circ} \mathrm{C}$, at which time $\mathrm{MgSO}_{4}$ was added and stirring was continued for another $10 \mathrm{~min}$. The entire solution was filtered through a cotton plug and washed with EtOAc. The eluant was concentrated to yield $251 \mathrm{~F}$ (90 mg, 86\% yield) as a white solid. $[\alpha]_{\mathrm{D}}-11.1$ (c 0.960 , $\left.\mathrm{CHCl}_{3}\right) ; \mathrm{mp}=110-112{ }^{\circ} \mathrm{C} ;{ }^{1} \mathrm{H} \mathrm{NMR}\left(400 \mathrm{MHz}, \mathrm{D}_{2} \mathrm{O}\right) \delta 0.79(\mathrm{~d}, J=6.3 \mathrm{~Hz}, 6 \mathrm{H}), 0.87(\mathrm{~d}$, $J=6.0 \mathrm{~Hz}, 3 \mathrm{H}), 0.95-1.23(\mathrm{~m}, 4 \mathrm{H}), 1.51-1.60(\mathrm{~m}, 2 \mathrm{H}), 1.68-1.77$ (m, 4H), 1.95-2.00 (m, $1 \mathrm{H}), 2.10(\mathrm{~d}, J=14.5 \mathrm{~Hz}, 1 \mathrm{H}), 2.49(\mathrm{t}, J=12.4 \mathrm{~Hz}, 1 \mathrm{H}), 2.54(\mathrm{t}, J=11.0 \mathrm{~Hz}, 1 \mathrm{H}), 3.09$ (dd, $J=3.3,13.5 \mathrm{~Hz}, 1 \mathrm{H}), 3.17(\mathrm{~d}, J=12.3 \mathrm{~Hz}, 1 \mathrm{H}), 3.30(\mathrm{~d}, J=13.5 \mathrm{~Hz}, 1 \mathrm{H}), 3.37$ (dd, $J=6.9,10.7 \mathrm{~Hz}, 1 \mathrm{H}), 3.54(\mathrm{dd}, J=4.4,10.9 \mathrm{~Hz}, 1 \mathrm{H}) ;{ }^{13} \mathrm{C} \mathrm{NMR}\left(100 \mathrm{MHz}, \mathrm{D}_{2} \mathrm{O}\right) \mathrm{d} 14.7$, 15.6, 17.5, 27.1, 28.3, 30.2, 31.4, 35.3, 37.6, 41.2, 44.3, 47.3, 53.1, 61.3, 64.8, 67.3; IR (neat) $3367,2800,2756 \mathrm{~cm}^{-1}$; MS (CI) m/e $252\left(\mathrm{M}^{+}+1\right)$; HRMS calcd for $\mathrm{C}_{16} \mathrm{H}_{30} \mathrm{NO}\left(\mathrm{M}^{+}\right.$ $+\mathrm{H}): 252.2327$, found 252.2317 . 


\section{References for Supporting Information}

(1) Evans, D. A.; Chapman, K. T.; Bisaha, J. J. Am. Chem. Soc. 1988, 110, $1238-1256$.

(2) Evans, D. A.; Bender, S. L.; Morris, J. J. Am. Chem. Soc. 1988, 110, 25062526. 

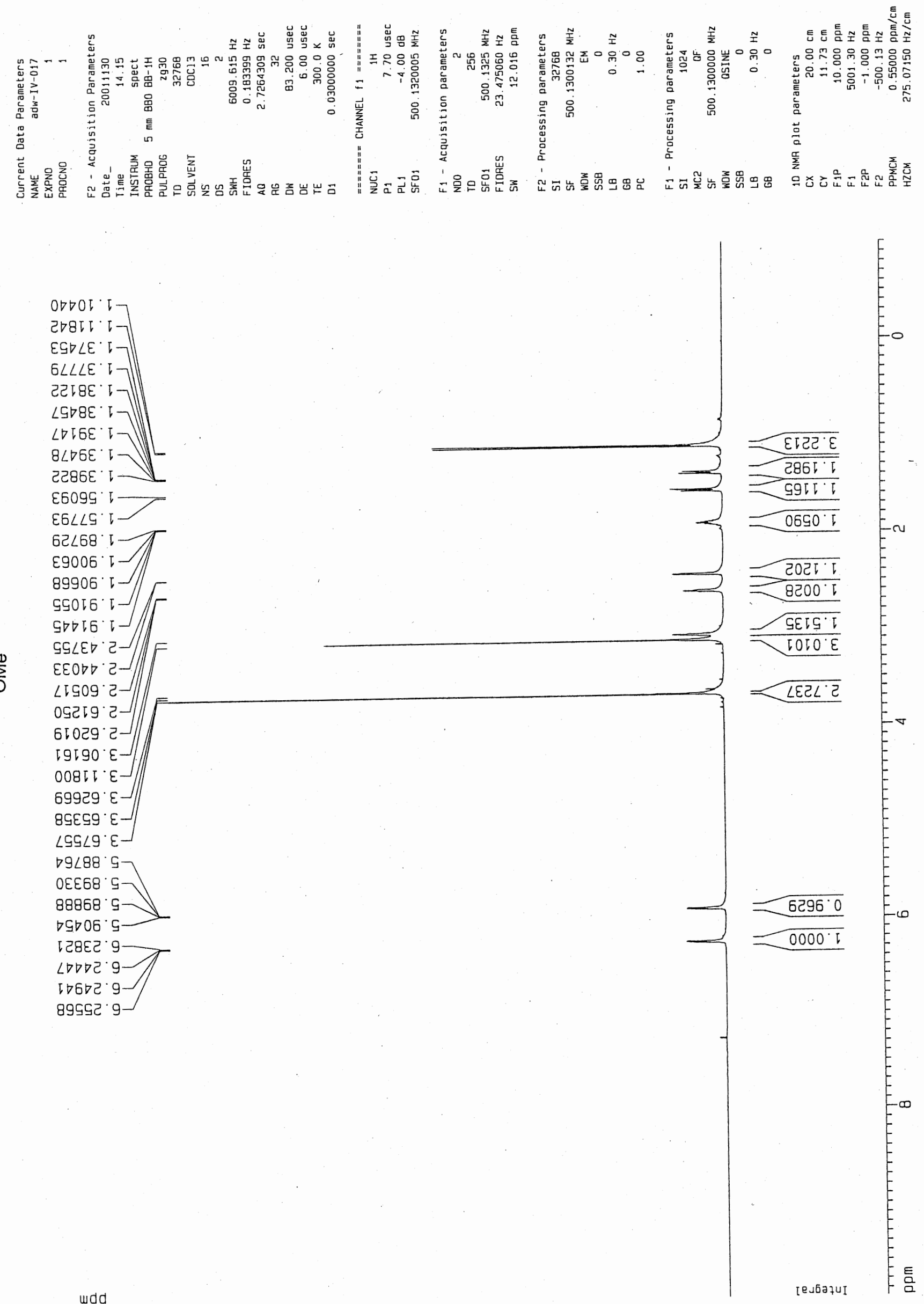


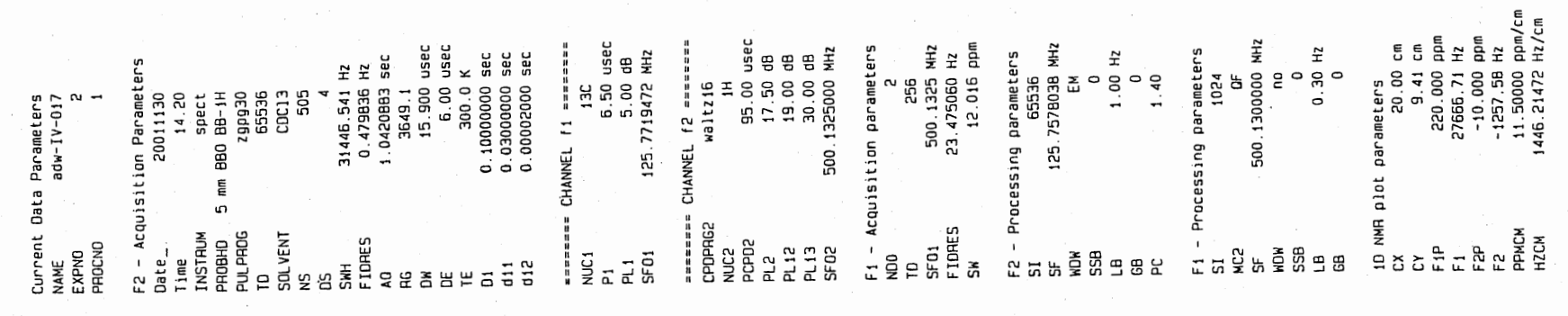

SOLOZ
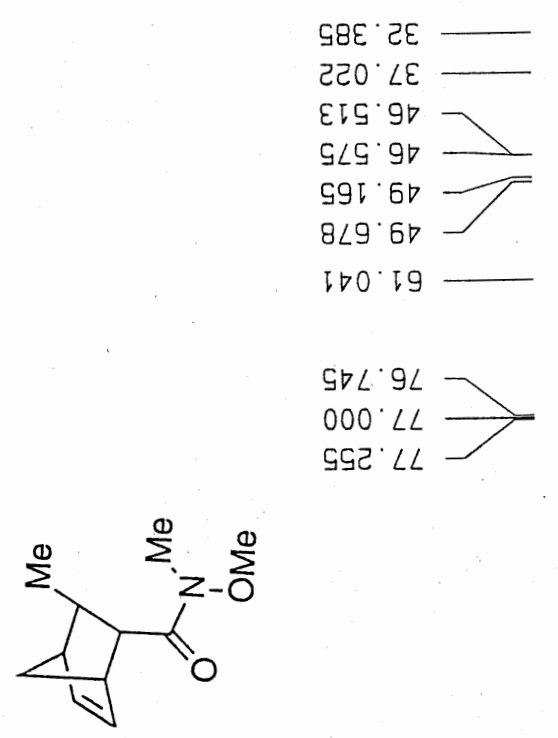

โโย' टE

SO6 $\angle E \downarrow$

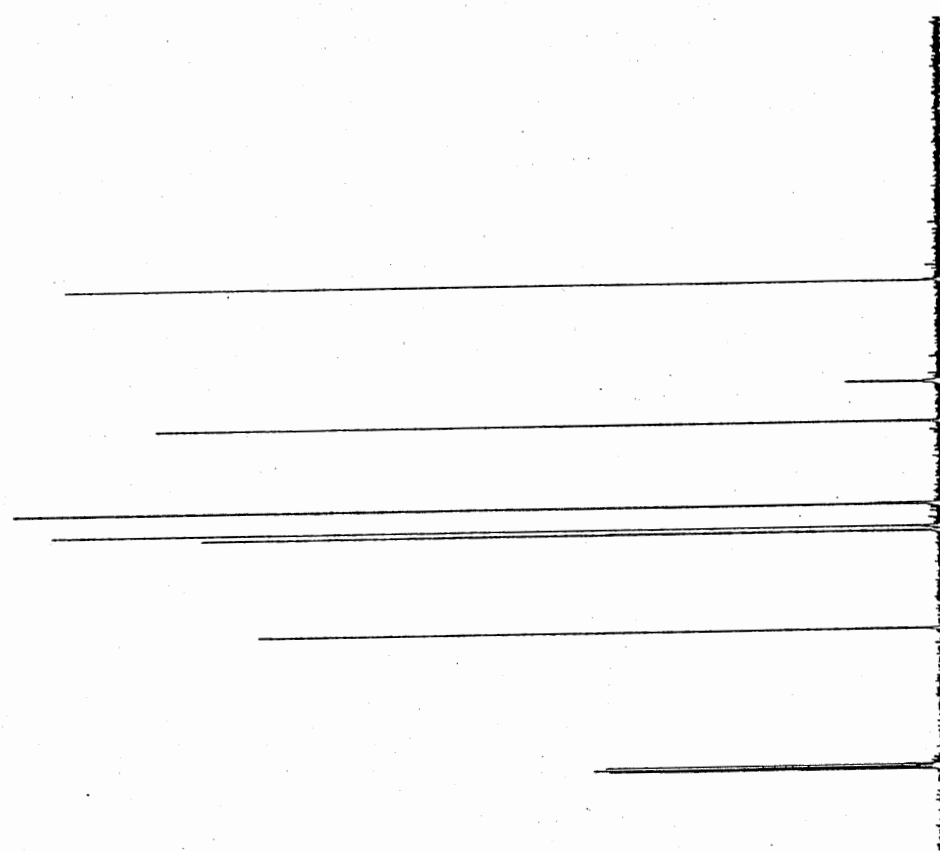

$$
\text { | }
$$



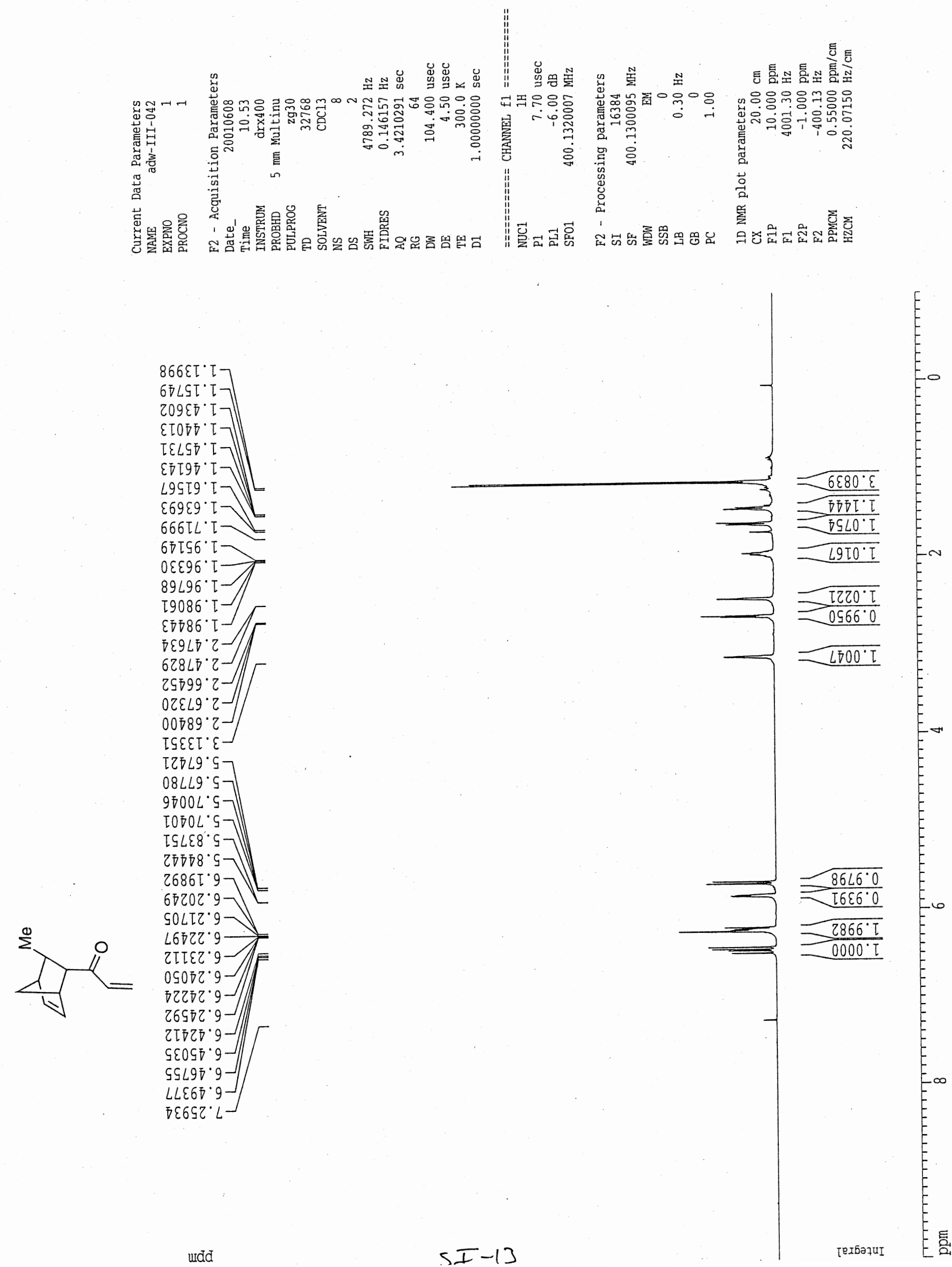

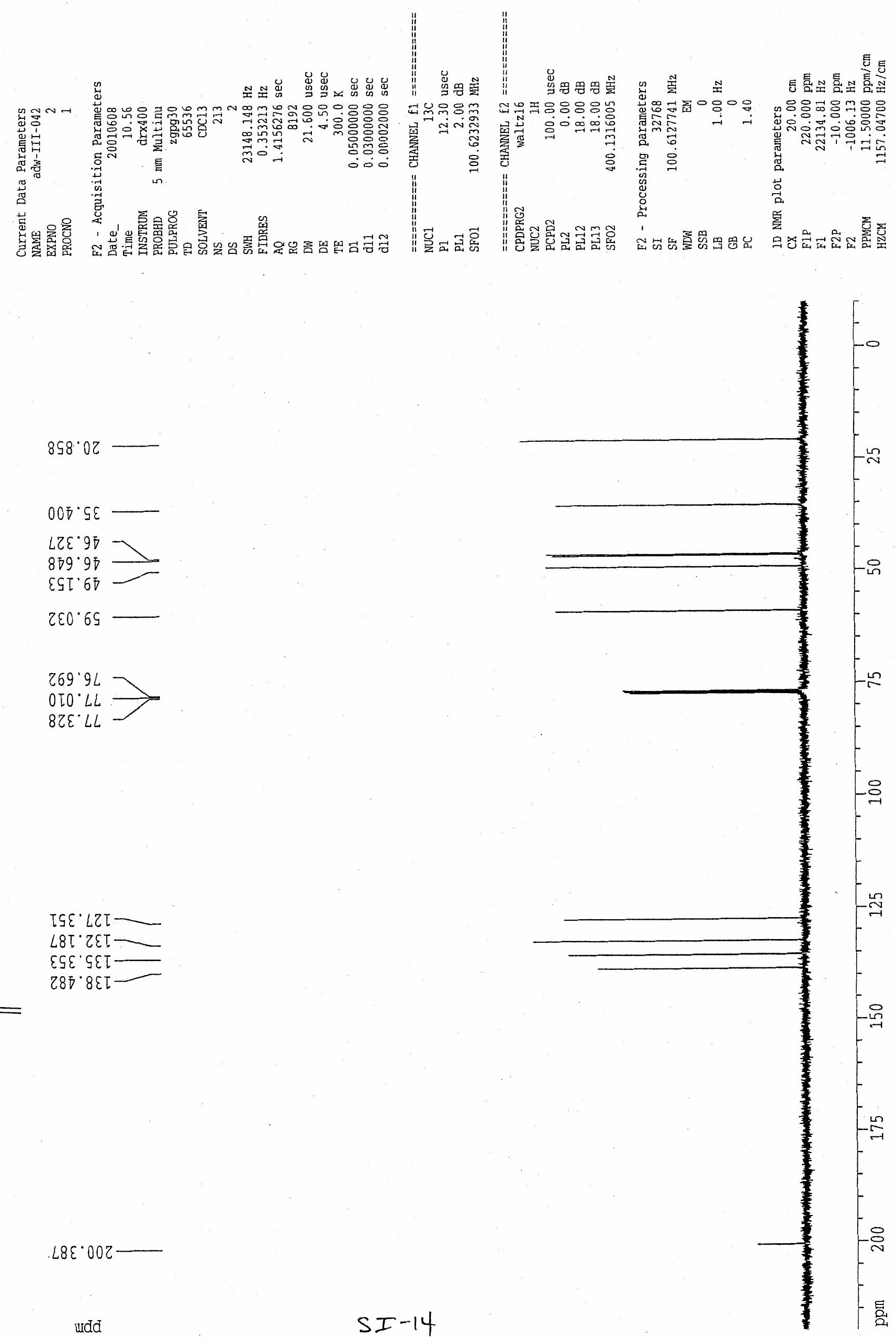

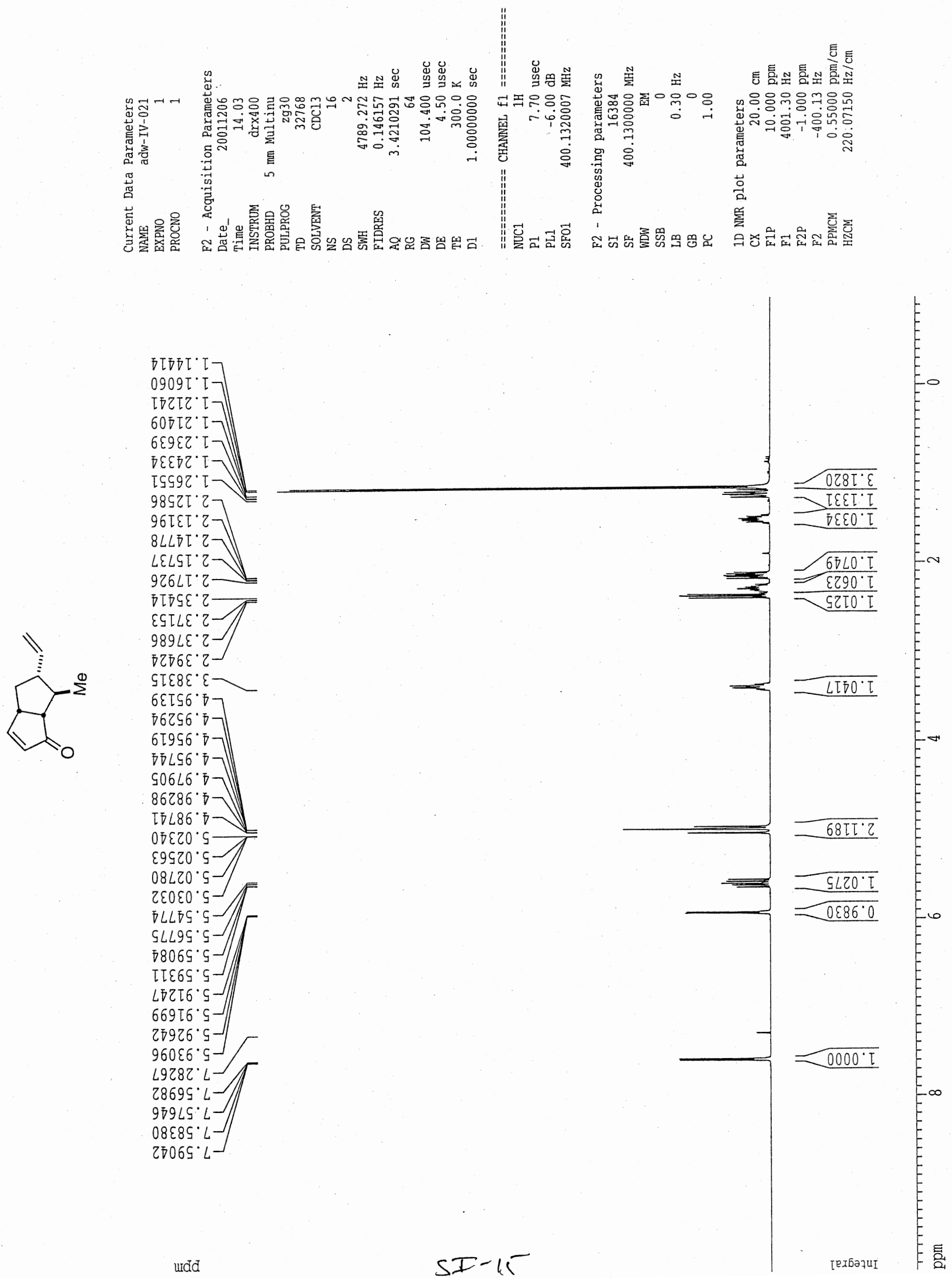


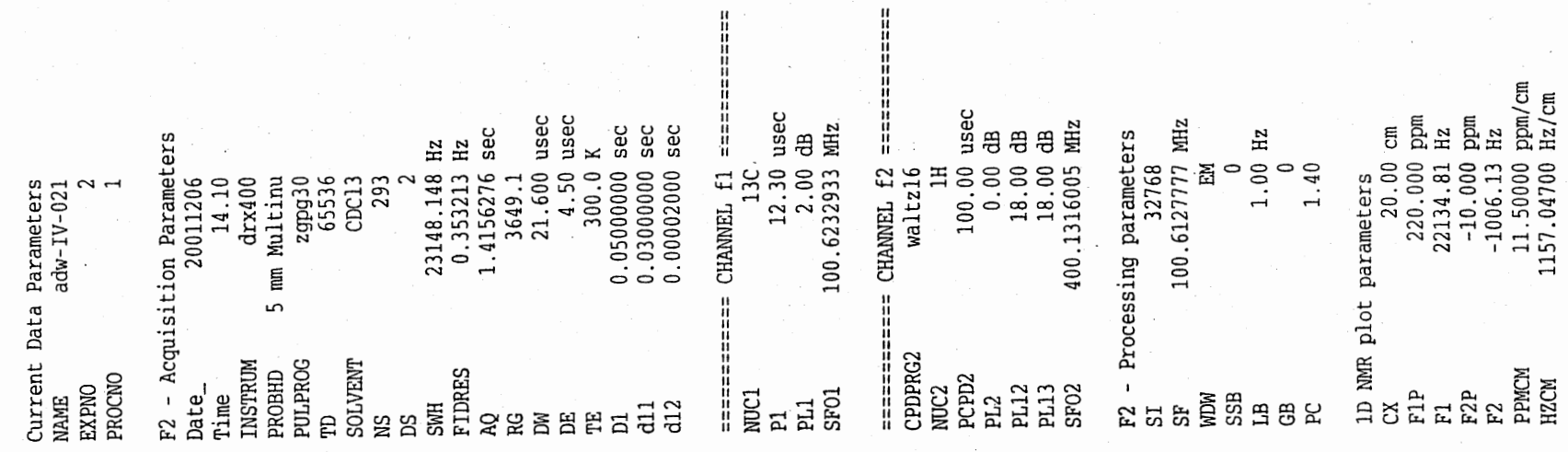

万ट๐.9

万IZ.

$697 \cdot 97$

Tt5.9S

$990^{\circ} \mathrm{LS}$
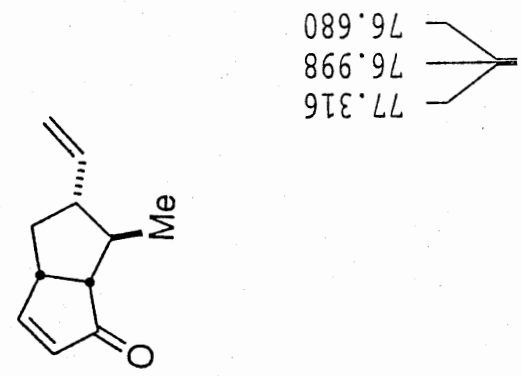

$9 T \varepsilon^{\circ} L L$

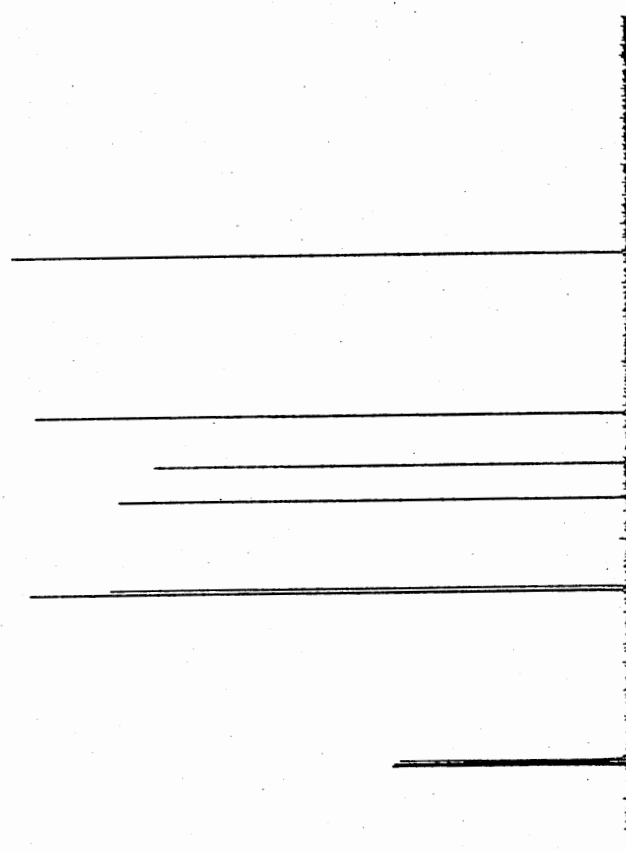

6SI'SIT-

9ST'TET-

$0 \angle 9^{\circ} 6 \varepsilon T$

$\angle 87^{\circ} 99 \tau$

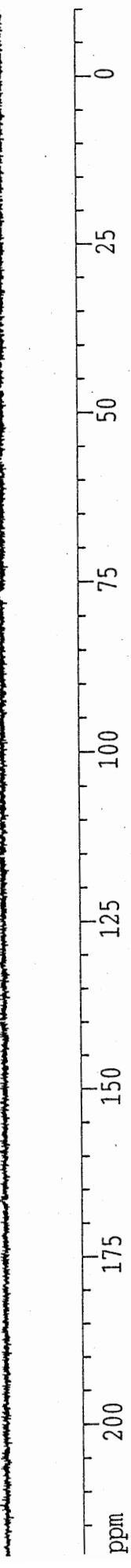




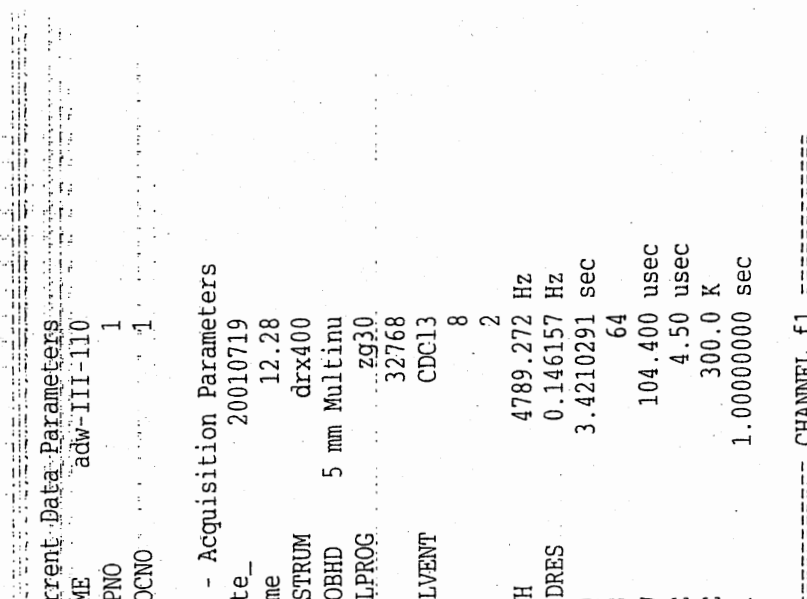

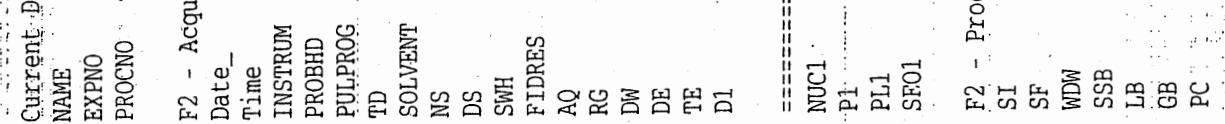

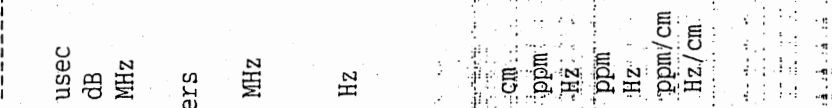

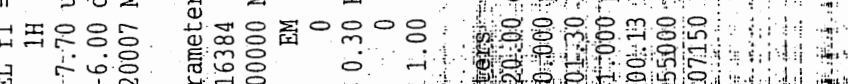

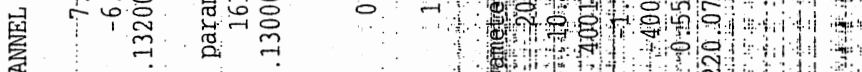

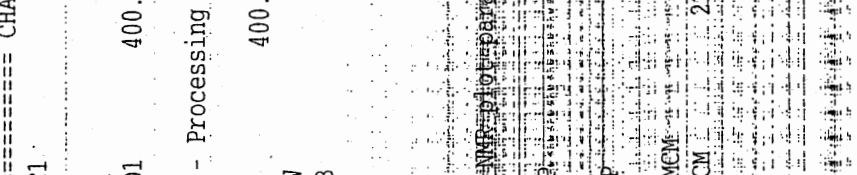

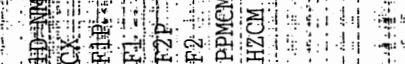

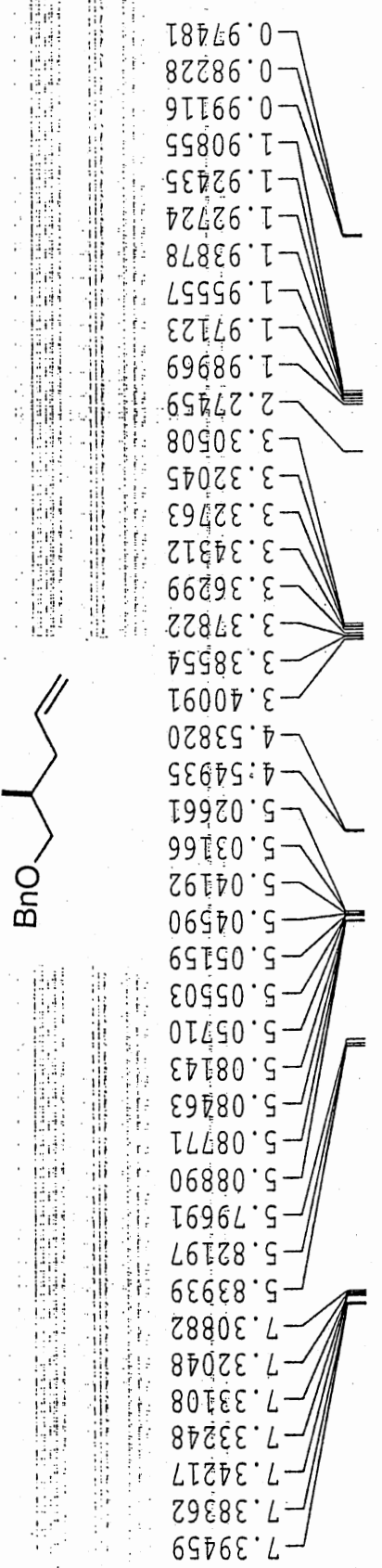

(1) (1) iptond m. (1)

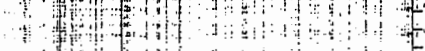



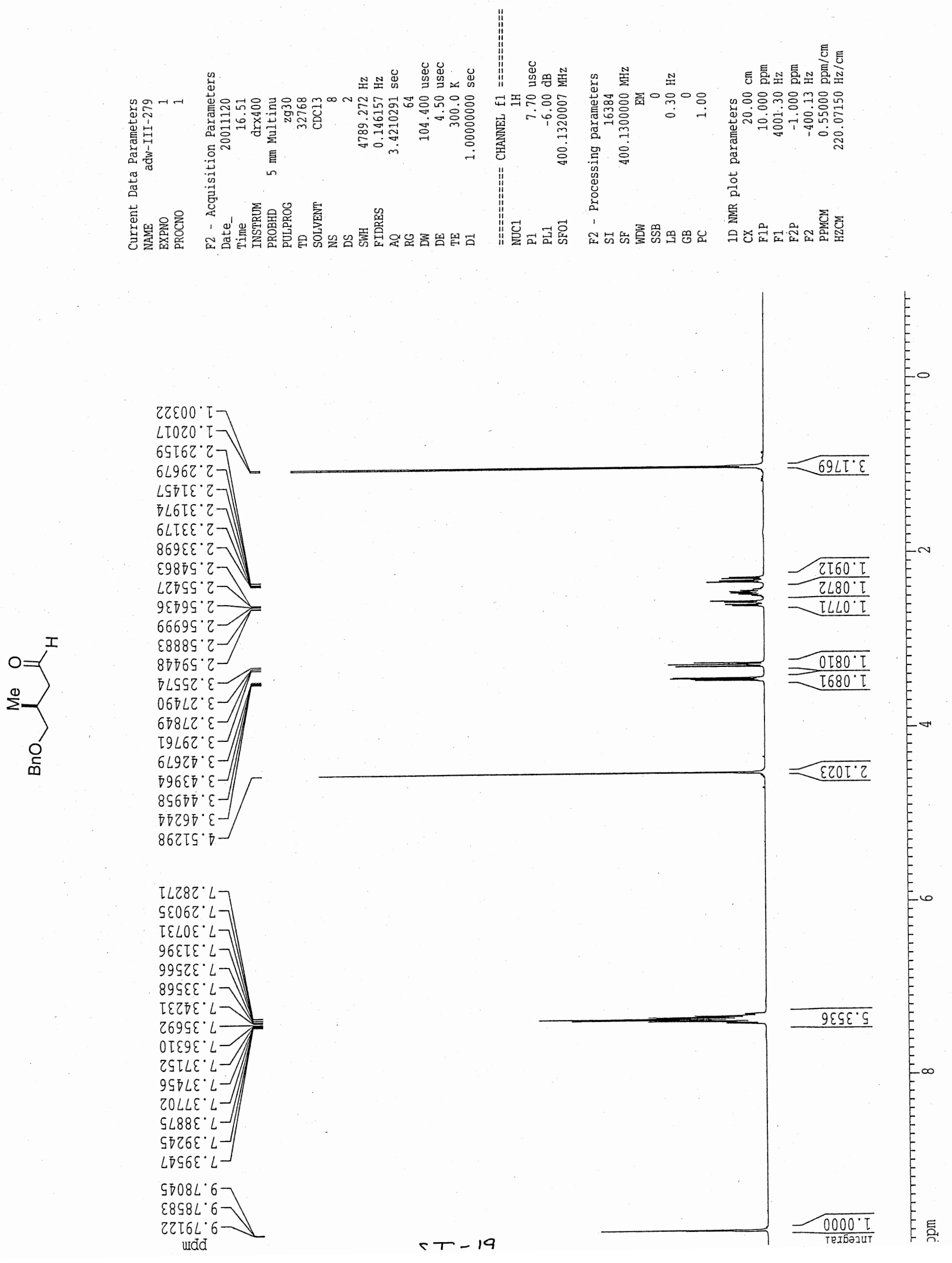

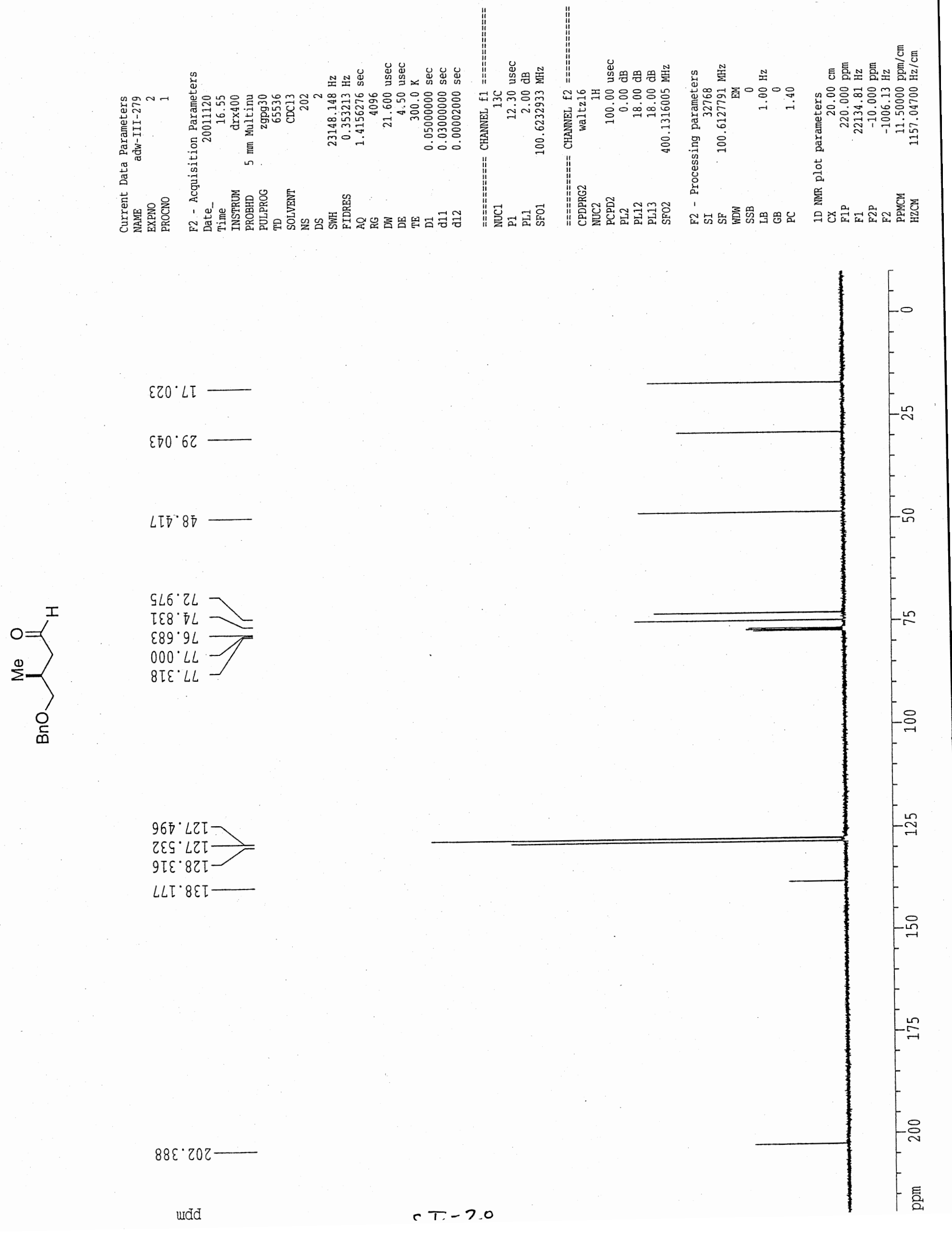

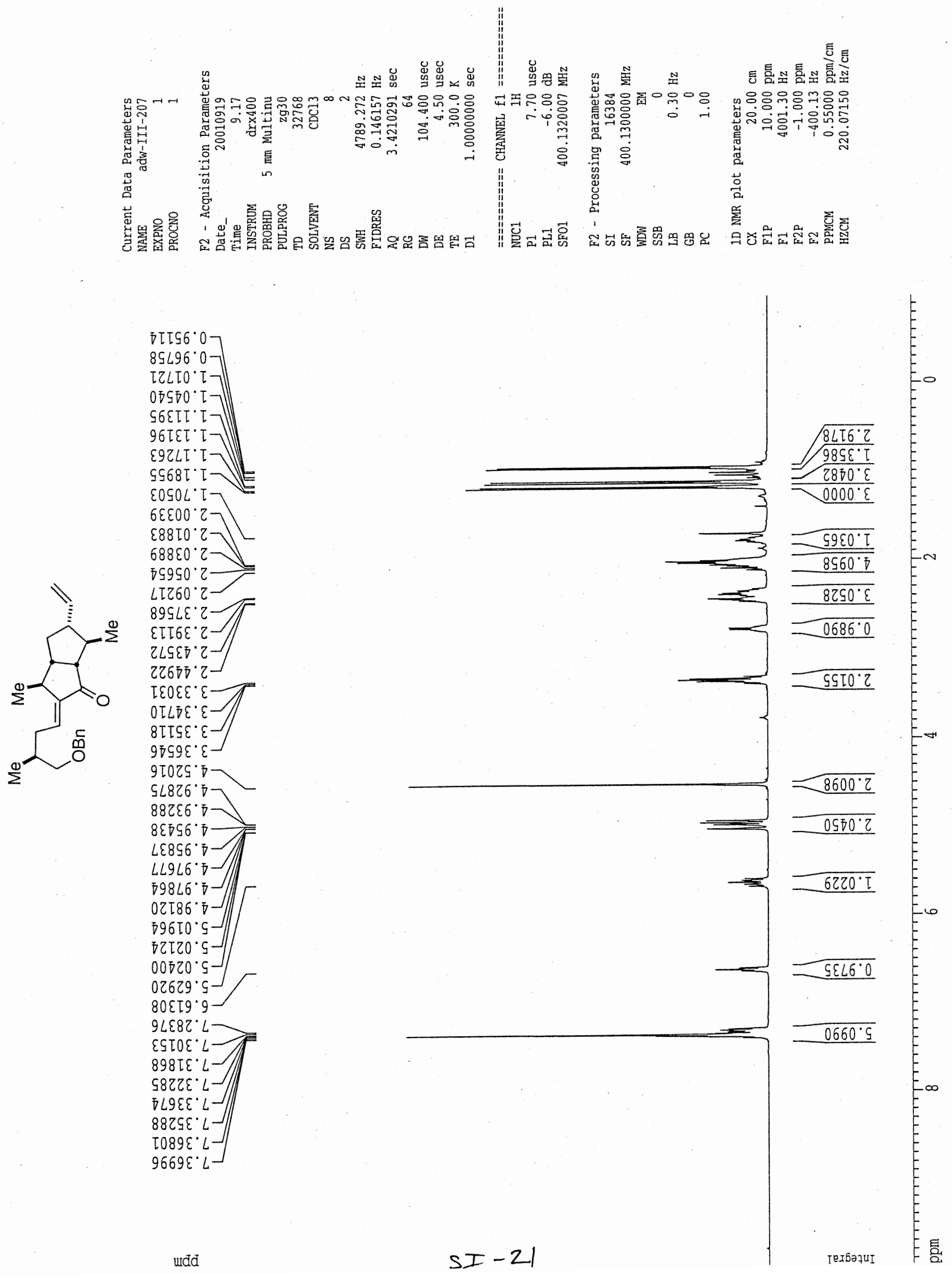

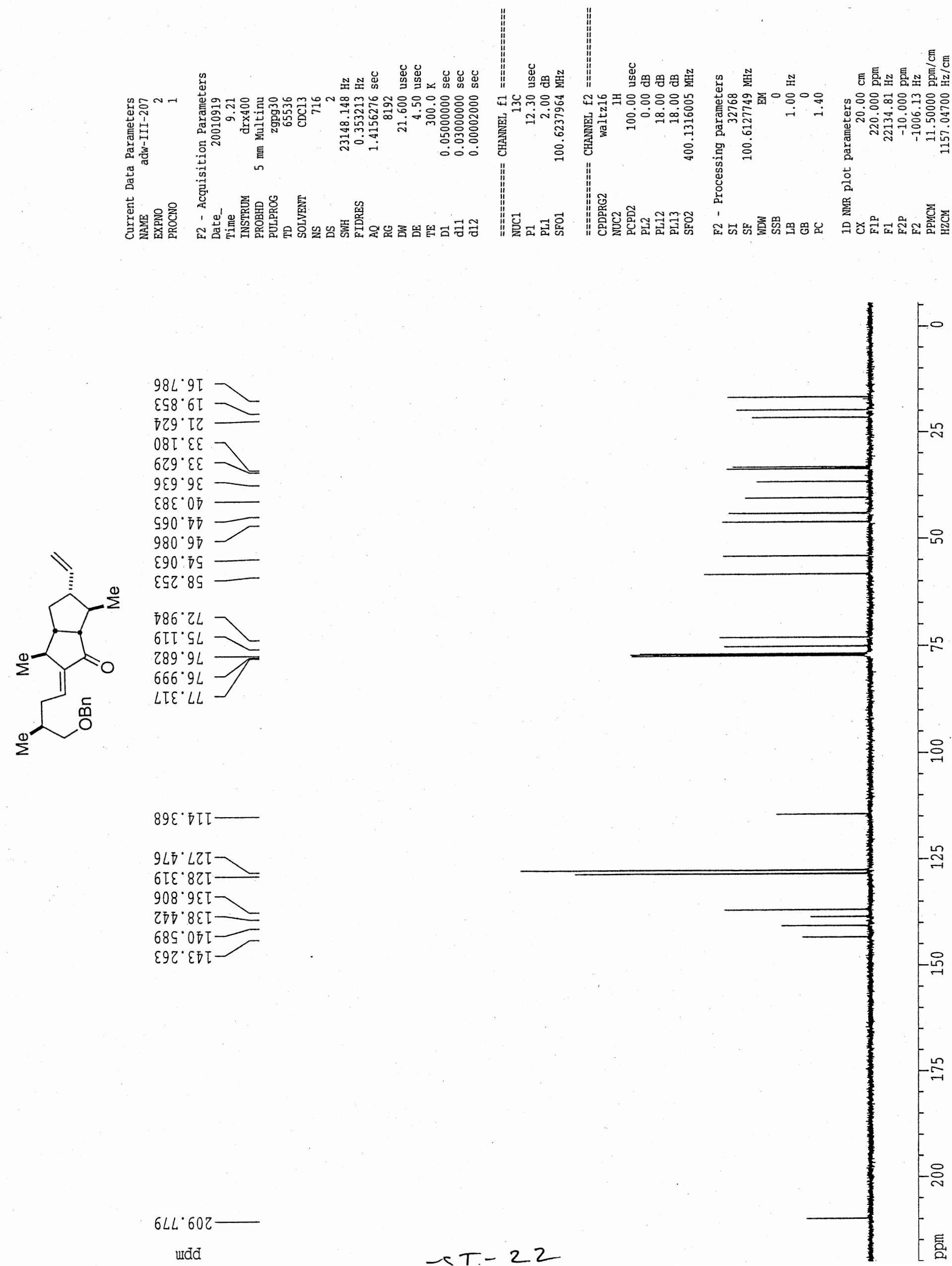

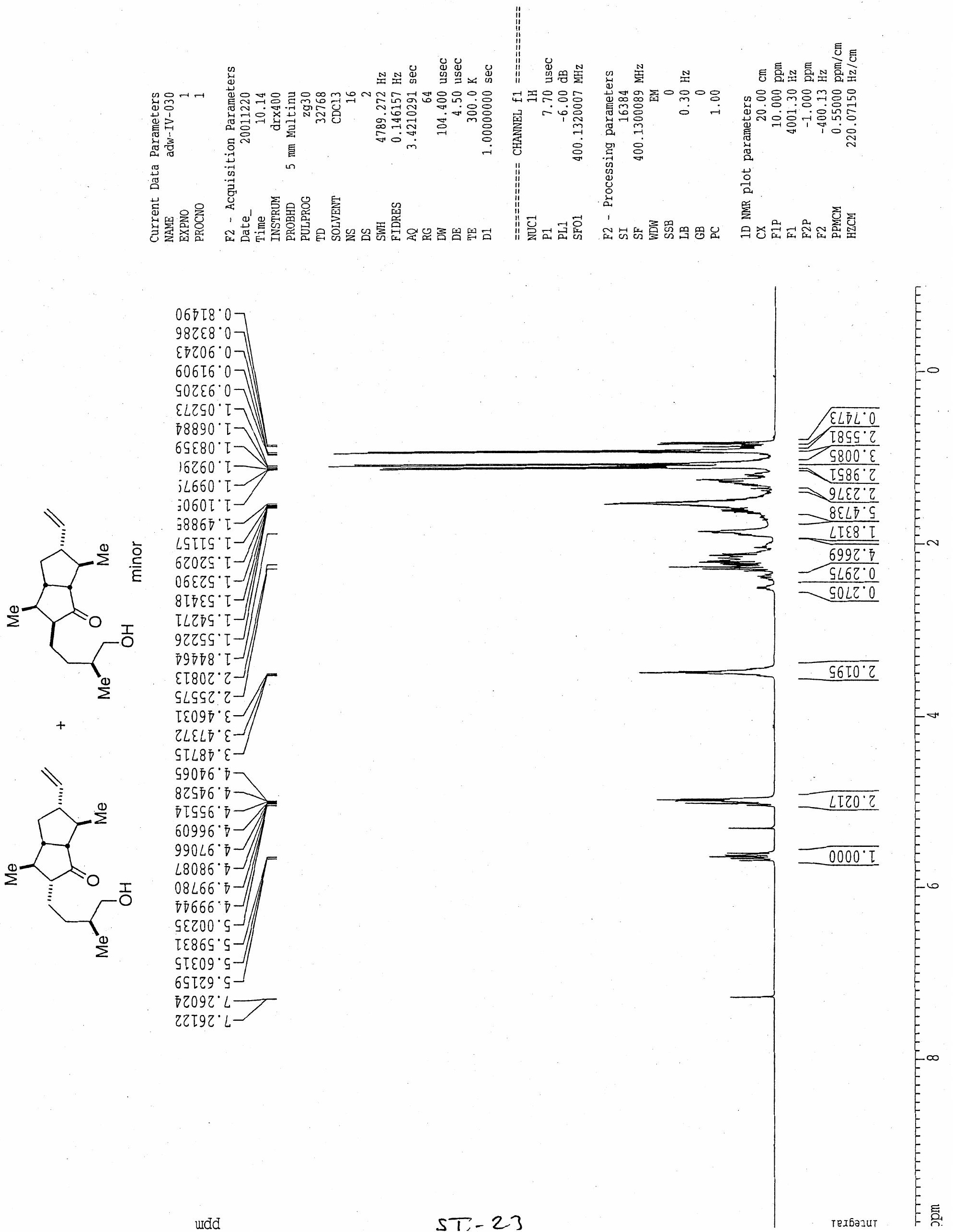

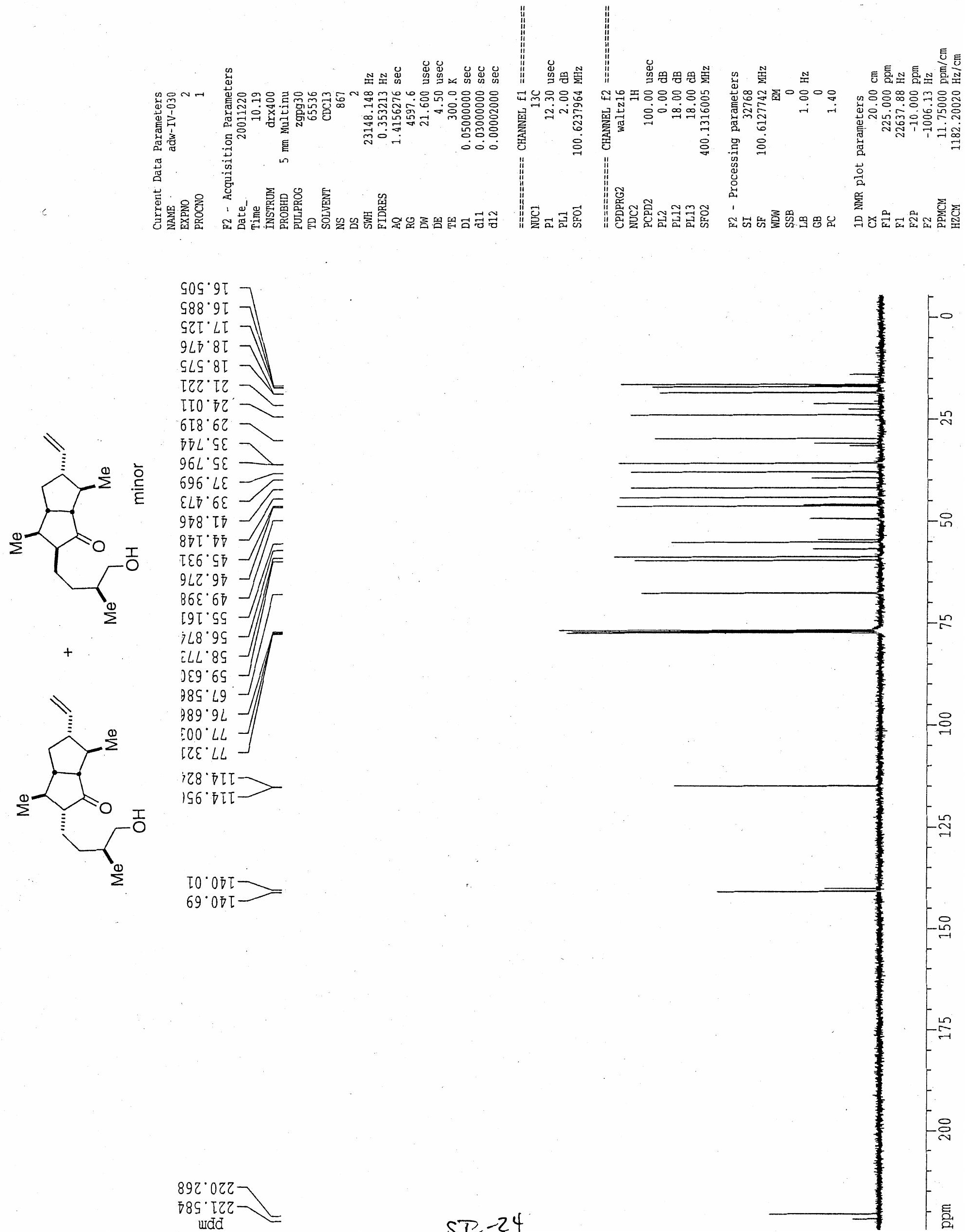

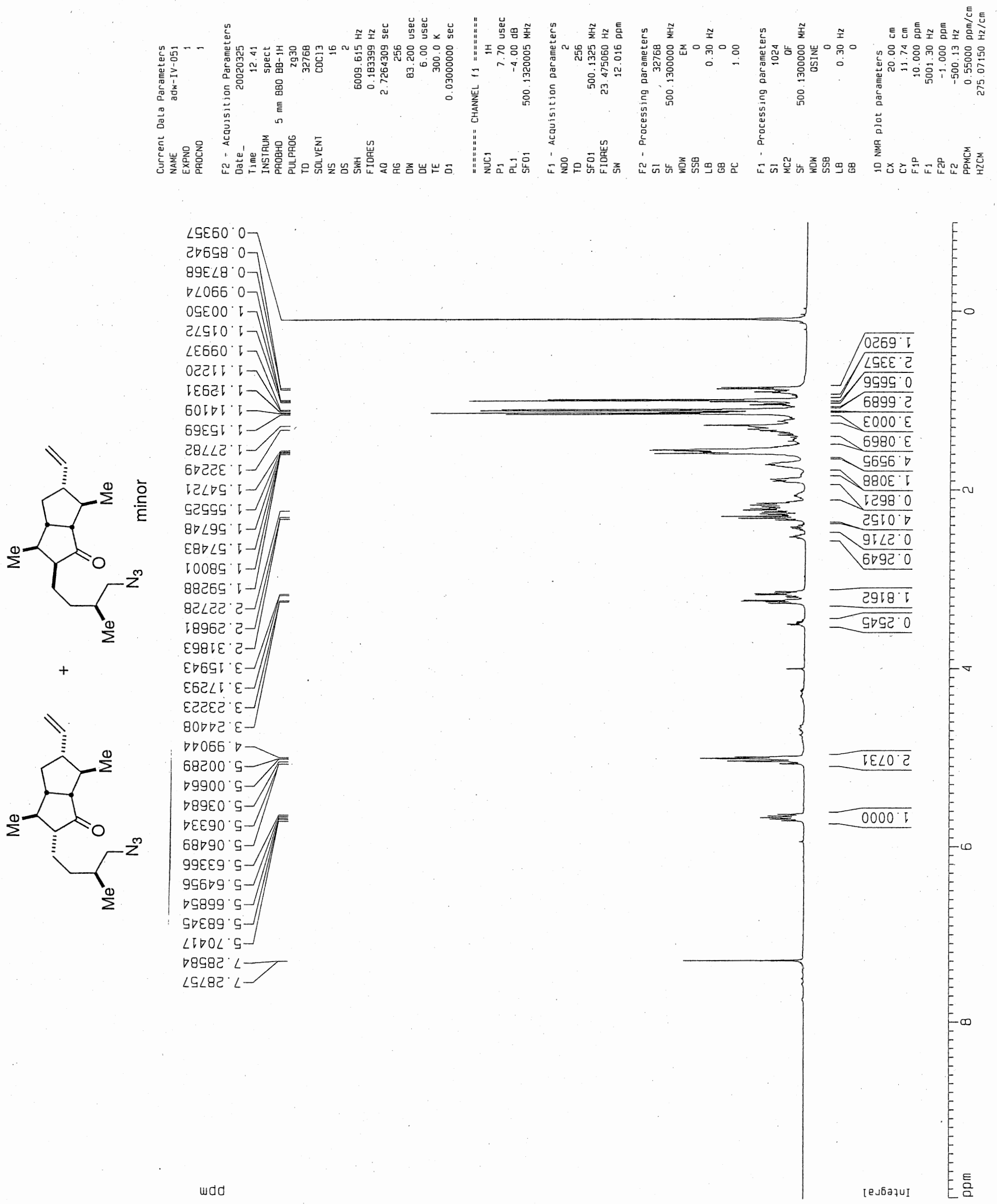

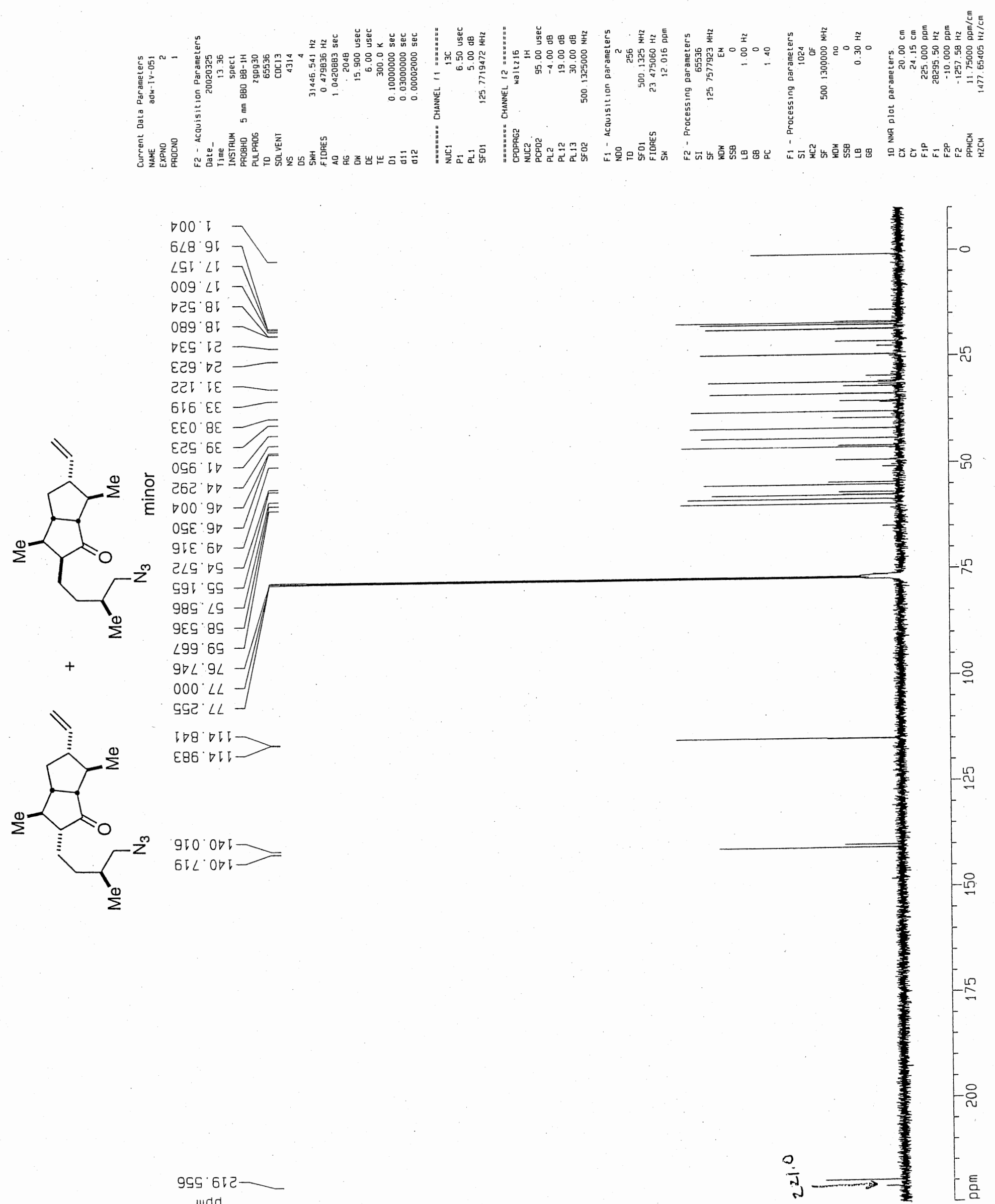

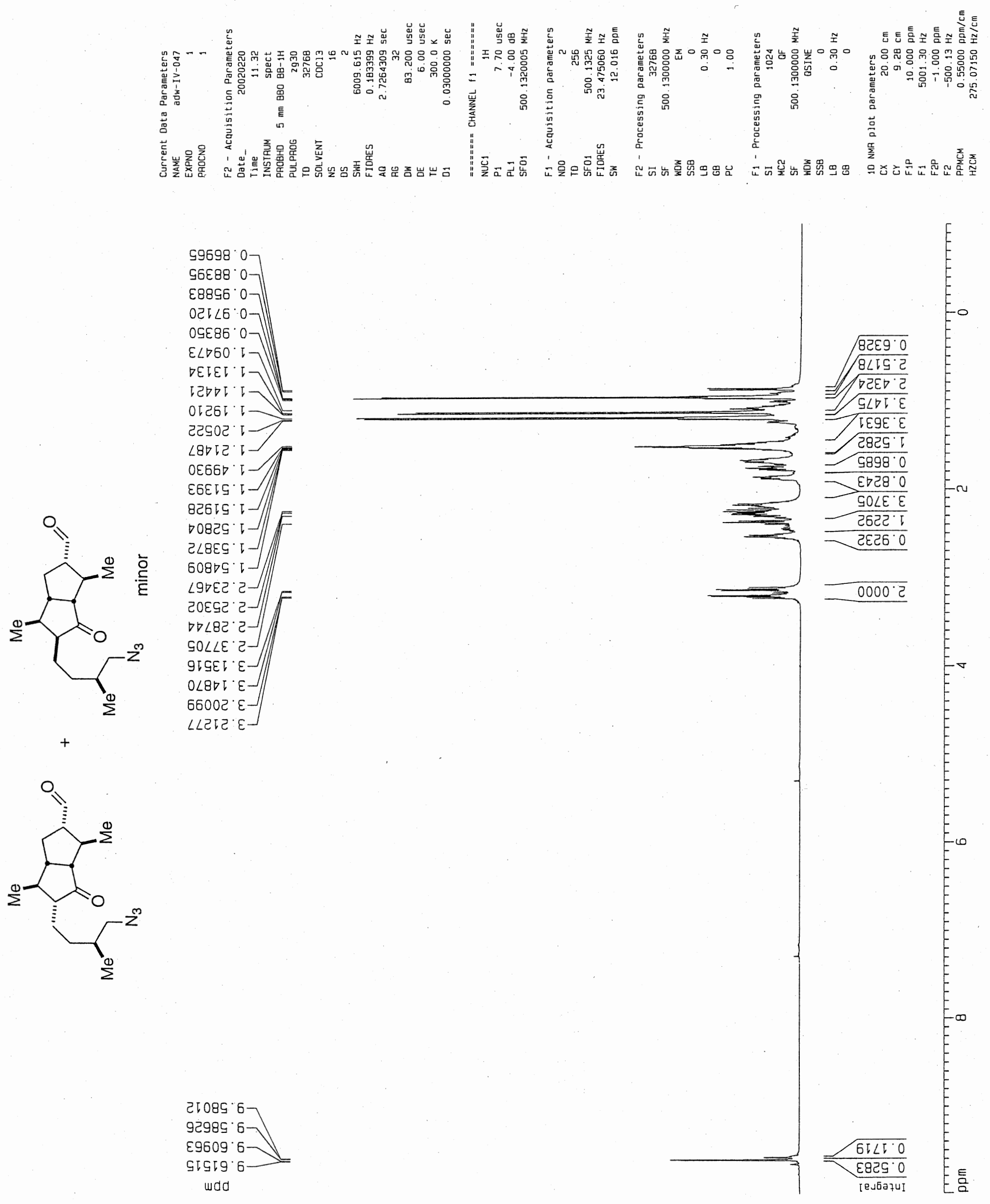

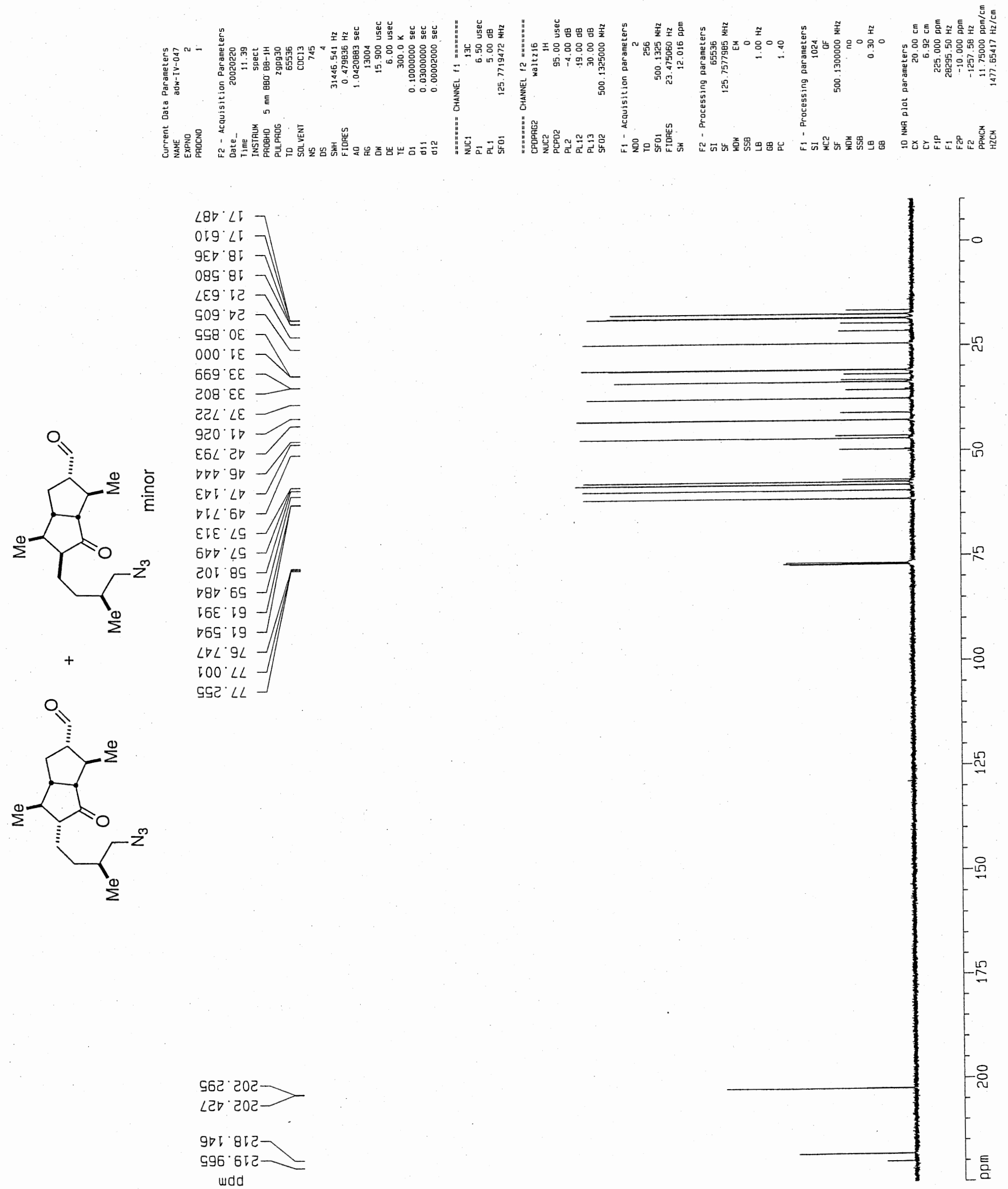

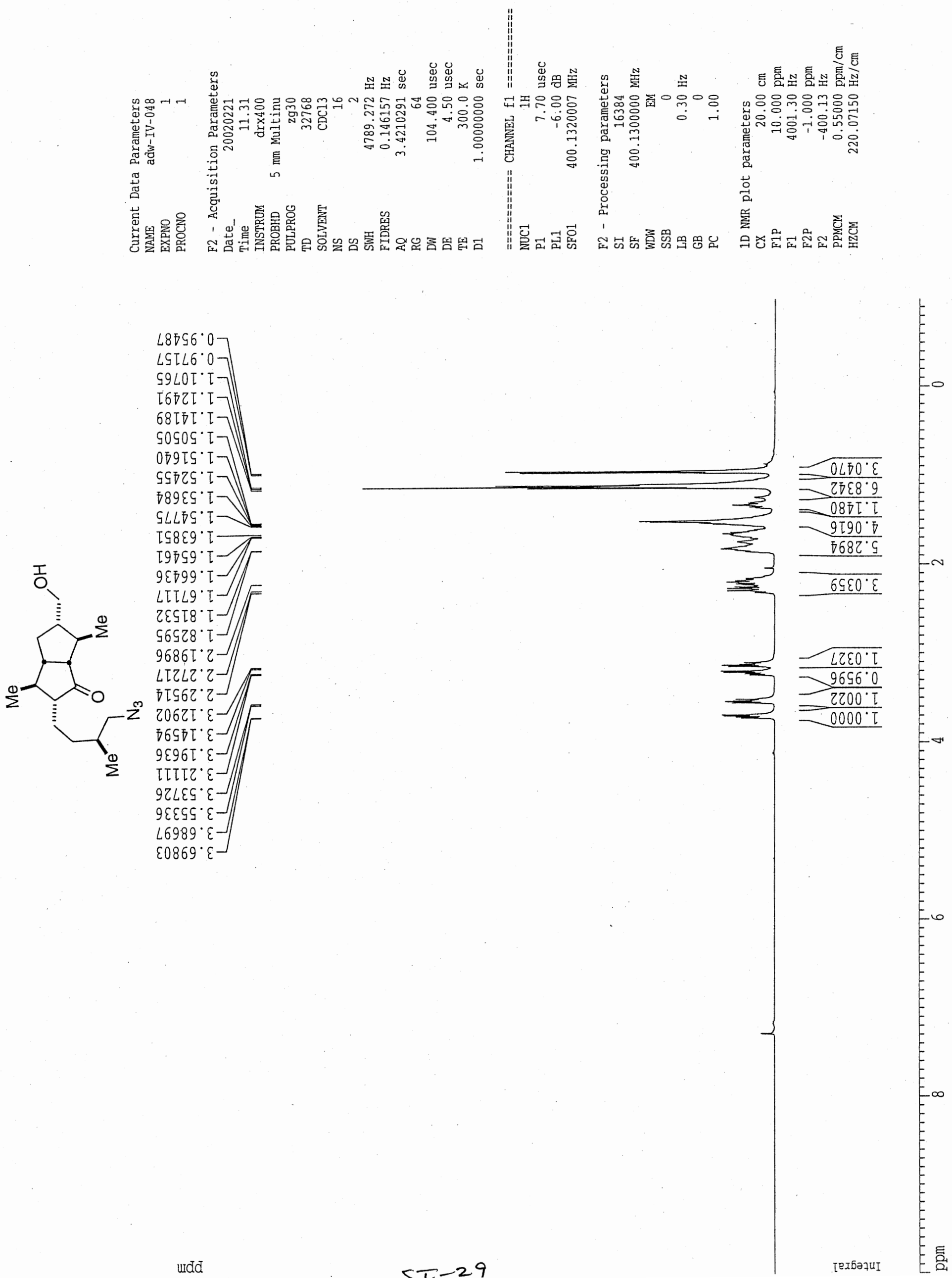

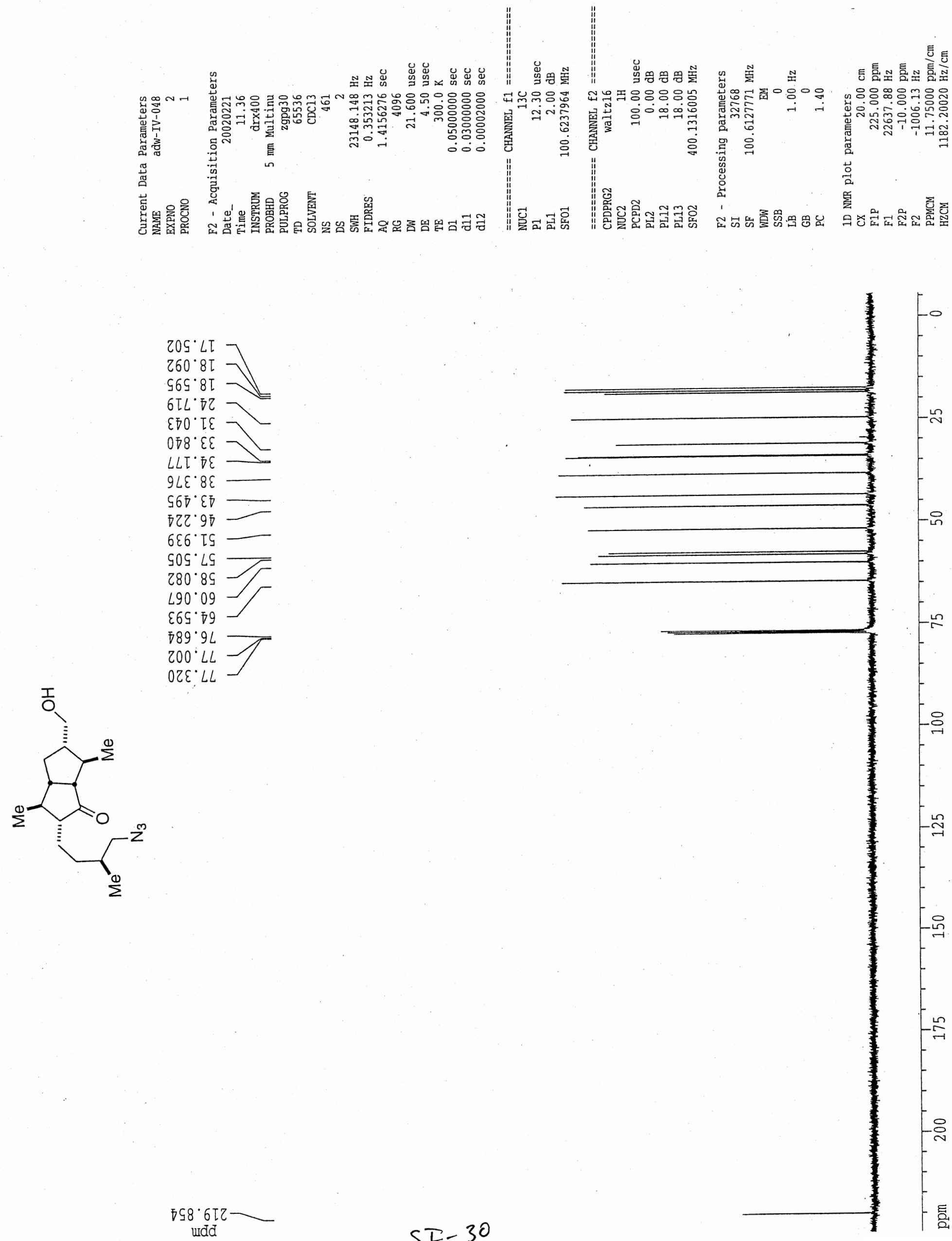

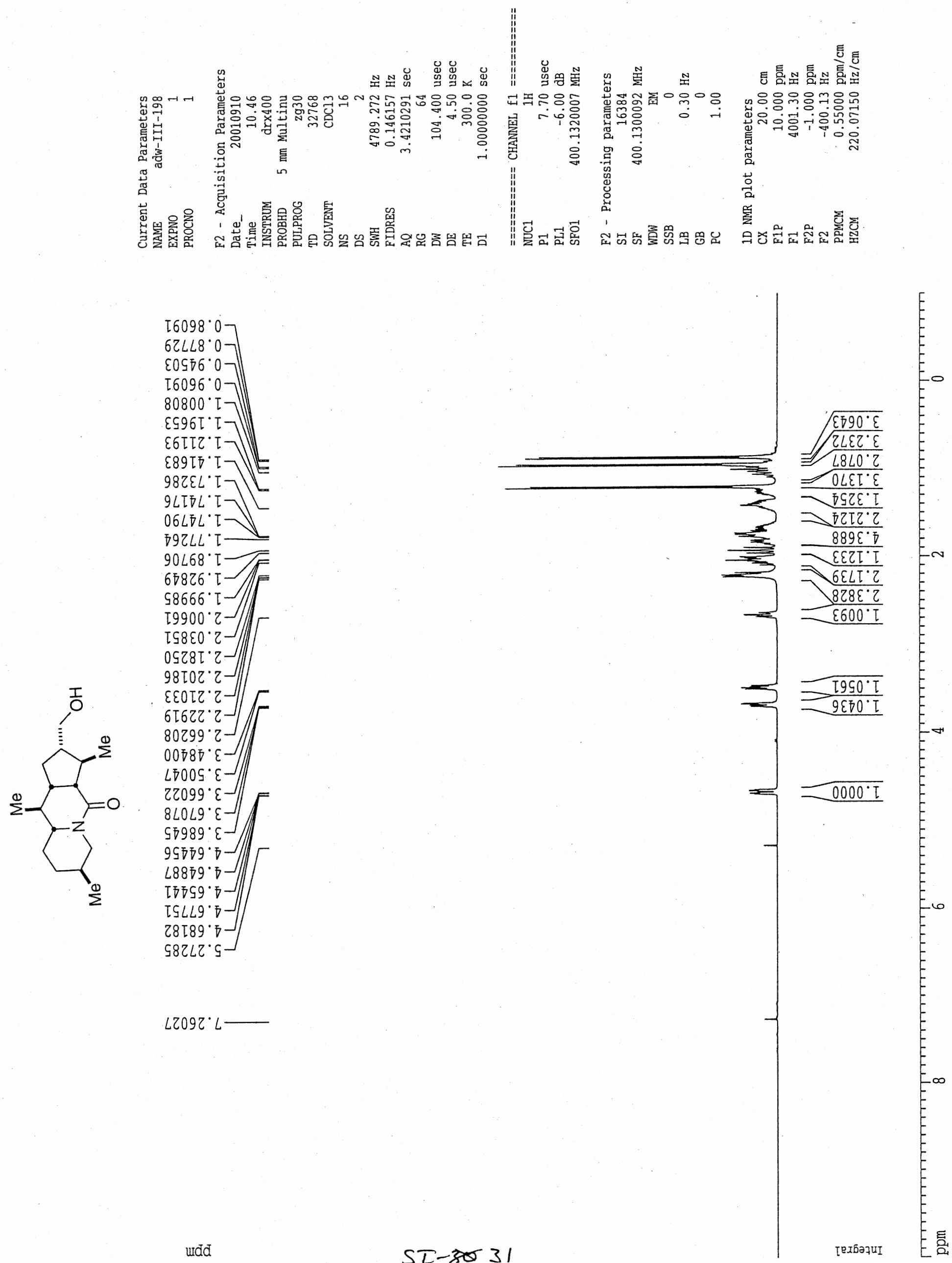

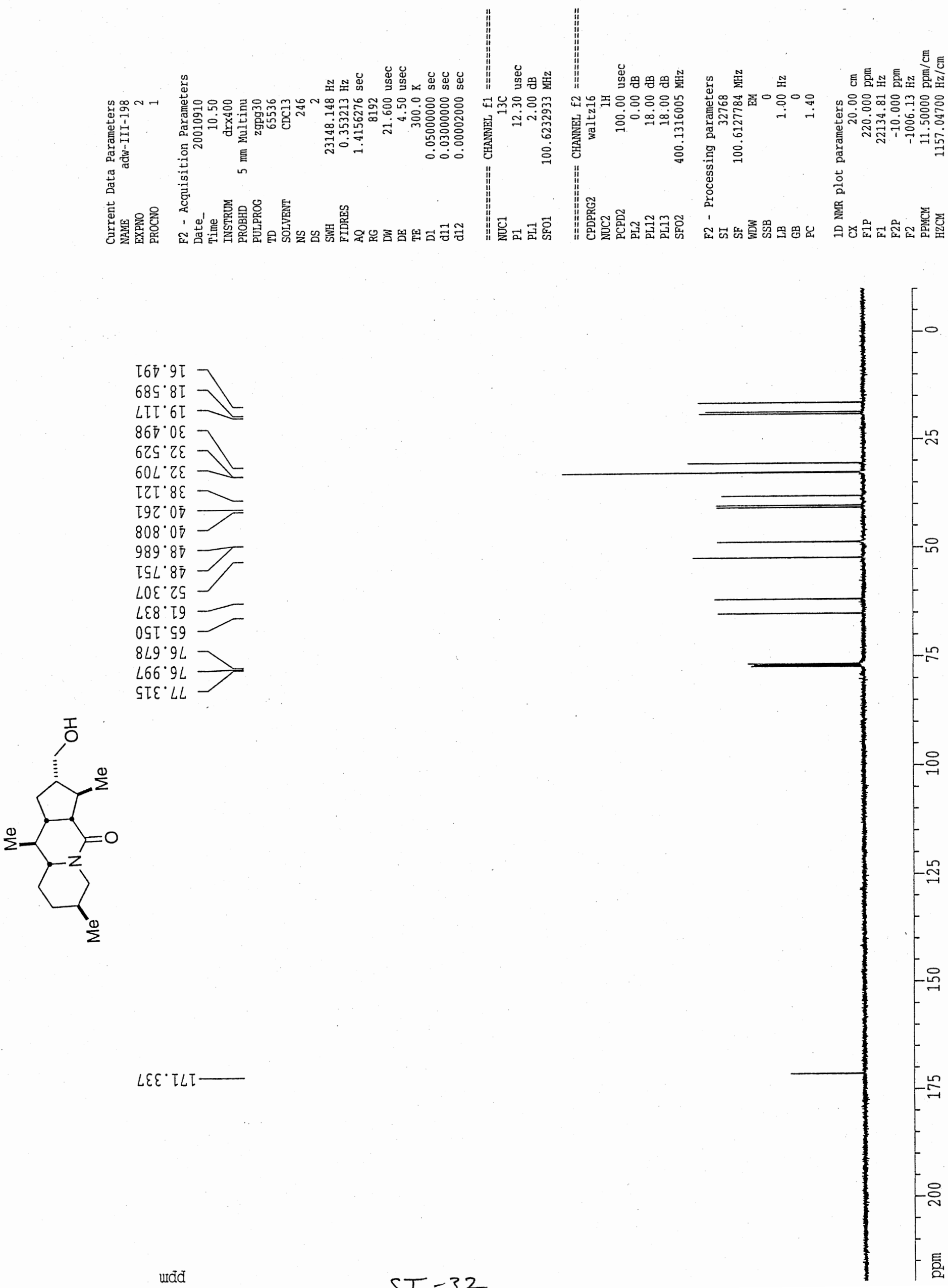

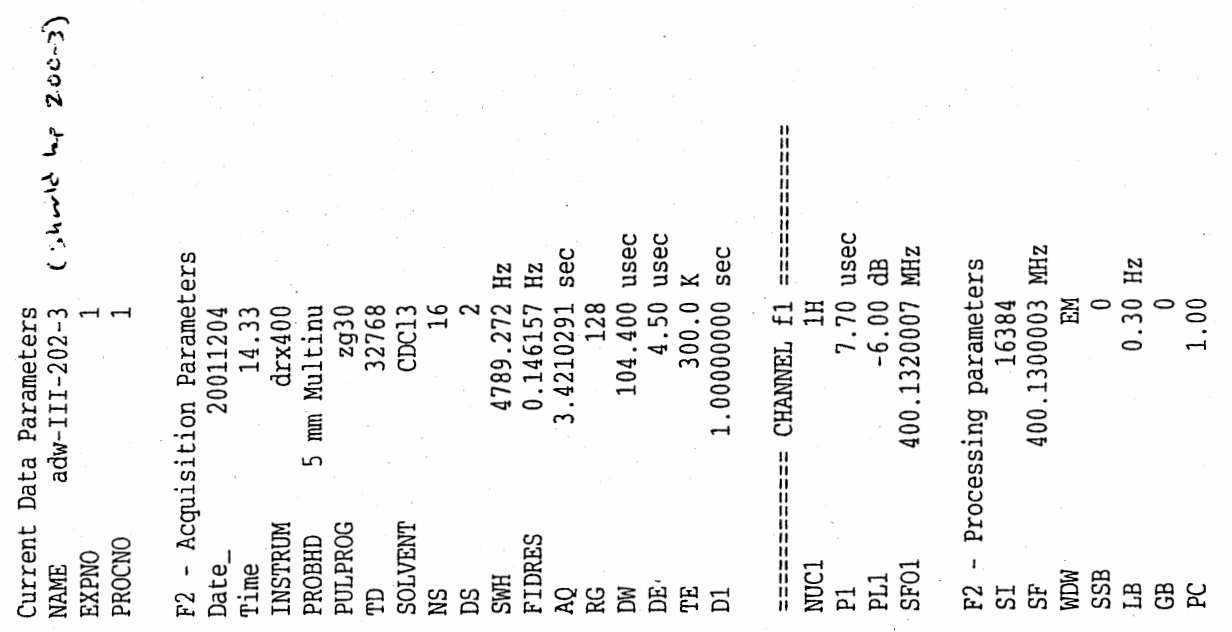

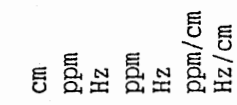

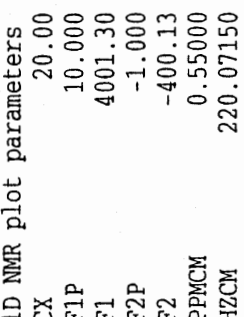

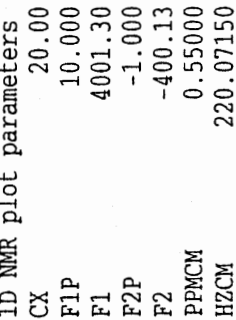
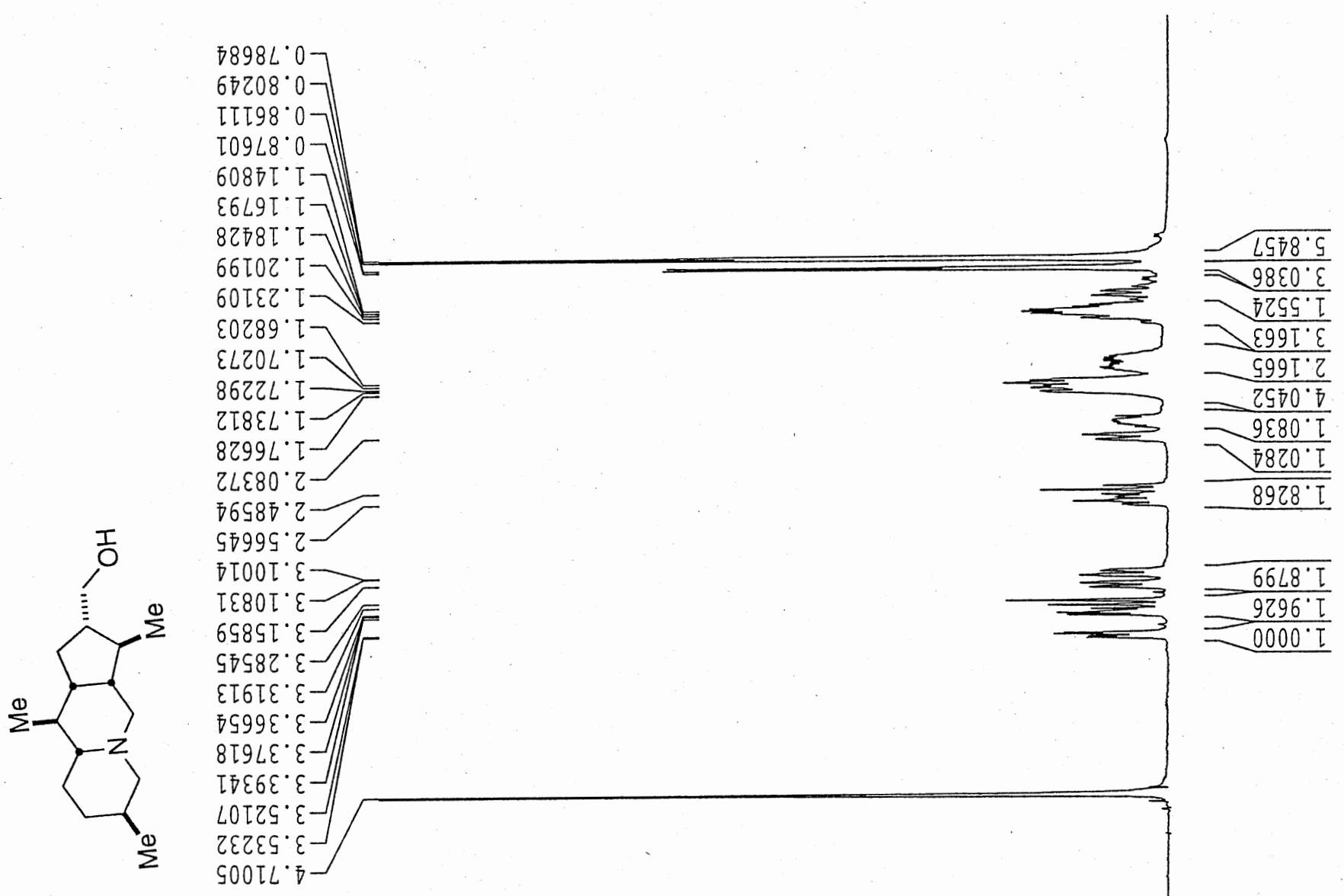

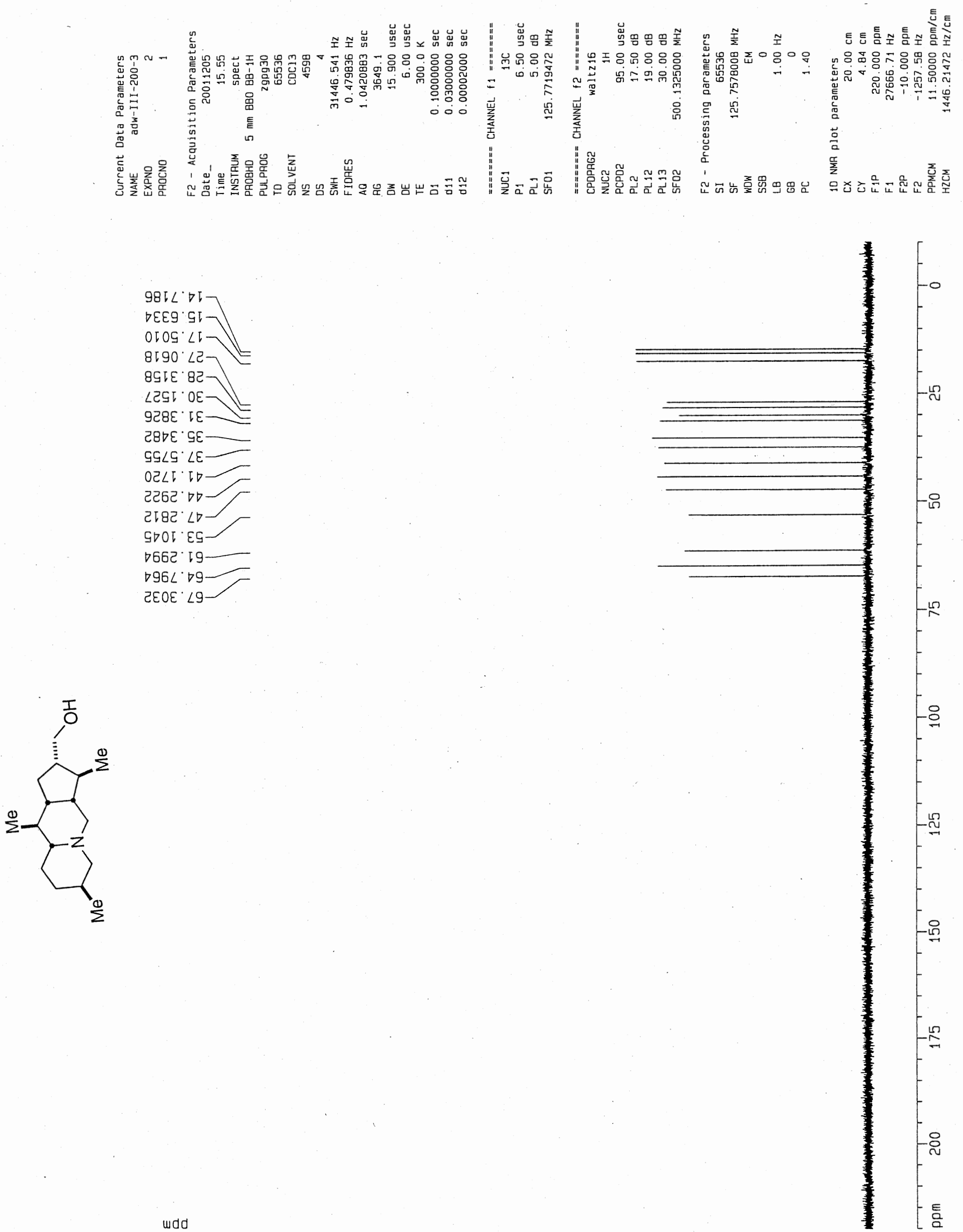